\title{
Hydrodynamic Design Study on Ship Bow and Stern Hull Form Synchronous Optimization Covering Whole Speeds Range
}

\author{
Yu Lu, ${ }^{1}$ Xin Chang $\left(\mathbb{D},{ }^{1}\right.$ Xunbin Yin, ${ }^{2}$ and Ziying $\mathrm{Li}^{1}$ \\ ${ }^{1}$ Naval Architecture and Ocean Engineering College, Dalian Maritime University, Dalian 116026, China \\ ${ }^{2}$ College of Shipbuilding Engineering, Harbin Engineering University, Harbin 150001, China \\ Correspondence should be addressed to Xin Chang; changxin_heu@outlook.com
}

Received 2 February 2019; Revised 31 May 2019; Accepted 13 June 2019; Published 8 August 2019

Academic Editor: Giorgio Besagni

Copyright (c) $2019 \mathrm{Yu} \mathrm{Lu}$ et al. This is an open access article distributed under the Creative Commons Attribution License, which permits unrestricted use, distribution, and reproduction in any medium, provided the original work is properly cited.

\begin{abstract}
The main objective of this article is to describe an innovative methodology of synchronous local optimization which considers the whole ship speed range being presented for a KRISO Container Ship (KCS). Parametric form approaches are adopted by employing a fairing B-spline curve in order to generate variants of the bow and stern of forms using form design parameters modified, resulting in an optimization system based on NSGA-II. The total resistance is calculated by the Rankine source panel method and the empirical formula which agrees well with the corresponding experimental data and further acquires validation with the overall error of $2.0 \%$. Accordingly, the ship forepart and stern form have been optimized under conditions of the single design speed and whole speeds range based on the considerations of generally distributed and variable operational speeds for the operating characteristics of modern container ships synchronously. The optimized result presents well-balanced drag reduction benefits which averagely remain above $4.0 \%$ of ship resistance decrease. Compared to the traditional optimization process which is based on a specific design speed, the newly developed method is more practical and effective in both automation and integration.
\end{abstract}

\section{Introduction}

Energy saving and low-carbon environmental protection are the two inevitable trends in shipbuilding industry. Meanwhile, the aims of ship energy saving and emission reduction have promoted the rapid development of the research in ship design and optimization. In recent years, driven by economic interests and environmental protection missions, domestic and international liner companies adopted new measures to slow down the ship's service speed; the benefits by doing so are even more remarkable to container ships.

The operation speed of modern container ship has been reduced year by year [1]. At the same time, the operating speed distribution is more decentralized and uniform. Due to the influence of the slow sailing strategy, the resistance performance of the single speed and the high speed container ships has been paid less and less attention. At the same time, the container ships designed for high speed have been subjected to great impact, because they cannot play a good resistance performance in the middle and low speed segment.
The present work presents an appropriate resistance optimization method of the bow and stern which considers whole speeds range, combining the parametric hull form transformation method, a RSM hydrodynamic solver, and modern optimization techniques. It should be noted that all the codes are in-house developed.

The methodology for hull form transformation based on the parametric ship-hull model is presented in Section 2. In 1950, Lackenby made an attempt to build the ship parametric model [2]. He obtained hull parametric variants through modifying the prismatic coefficient, the center of buoyancy, and the extent and position of parallel mid-body. Lackenby transform is applied to optimization of ship design by Harries [3], who completed the ship-hull transformation by controlling the transverse section area curve containing the design parameters; see, e.g., Harries and Abt [4], Nowacki and Kaklis [5], Harries and Nowacki [6], Lee [7], Maisonneuve et al. [8], Saha, Suzuki, and Kai [9, 10], Kim [11], Pérez and Suárez [12-14], Abt and Harries [15], Harries [16], Zhang and Zhu [17], Tahara, Peri, Campana, and Stern [18], and A.I. 
Ginnis and P.D. Kaklis et al. [19-22]. In this work, the method is concerned for the modification and reconstruction of the hull bow and stern form using a fairing B-Spline parametric curve.

Section 3 is devoted to the prediction method of the total resistance performance, which has been defined as the optimization objective function in this work. The ITTC 1957 model-ship correlation formula and the Rankine source panel method are applied to calculate the frictional resistance and wave-making resistance, respectively. The Rankine sources method is based on the potential-flow without considering viscosity. Nevertheless, for a sophisticated shiphull optimization system, it is more simple and convenient and has been proved to be accurate, reliable, and efficient, so it has been investigated in various studies (Abt and Harries et al. [23], Lu, Chang, and Hu [24], Lowe and Steel [25], Suzuki, Kai, and Kashiwabara [26], Zhang, Kun, and Ji [27], Zhang [28], Chi, Huang, and Noblesse [29], Choi, Park, and Choi [30], and Chi, Huang, and Kim [31]).

Section 4 presents the Fast Elitist Nondominated Sorting Genetic Algorithm II (NSGA-II) used to search for the minimum ship total resistance for the ship-hull optimization. NSGA-II is a kind of modified GAs with low computational requirements. In 1975, Holland [32], a professor of Michigan State University, firstly proposed Genetic Algorithm (GA), which is a computational model simulating evolutionary process in nature. Deb et al. improved GA and proposed NSGAII $[33,34]$ with Fast nondominated sorting method proposed to reduce the computational difficulty, the evaluation, and comparison of congestion put forward to help to make quick judgment for the same level of individual behavior and elitist strategy introduced to expand the space sampling.

Finally, two optimization cases for KCS [35-37] are set up and presented in Section 5. The first case deals with a ship-hull optimization at design speed, while the second case involves a total resistance minimization problem against multiple objective functions covering all speed range.

\section{Hull Form Transformation Method}

2.1. Definition of Fairing B-Spline Parametric Curve. In this article, the hull form transformation is realized by the fairing B-spline parametric curve transformation. The deformation of B-spline parametric curves is realized by adjusting the input characteristic parameters, and then the deformation of the characteristic parameter curve is mapped to the geometric deformation of the hull.

The fairing B-spline parametric curve is formed by applying the constraint conditions on a B-spline curve. The spatial spline curves parameterized by $u$ are expressed by $\boldsymbol{r}(u)$ :

$$
\boldsymbol{r}(u)=(x(u), y(u), z(u))
$$

The equation corresponding to the curve with $\mathrm{mth}$ fairness can be expressed as

$$
L_{m}=\int_{0}^{1}\left(D^{m} \boldsymbol{r}(u)\right)^{2}, \quad \text { where } D^{m}=\frac{d^{m}}{d u^{m}}
$$

Some control constraints should be satisfied in the fairing process.

(1) Distance Constraint Condition. The Euler formula is used to express the distance between the control point $P_{i}$ and the spline curve $\boldsymbol{r}(t)$. The distance shall meet the following condition:

$$
A=\sum_{i=0}^{n}\left[w_{i}\left(\boldsymbol{r}\left(u_{i}\right)-P_{i}\right)\right]^{2} \leq \varepsilon
$$

where $w_{i}$ is distance weight and $\varepsilon$ is a tolerance greater than zero.

(2) Curve Ends Constraint Condition. The tangent slope and curvature of the starting point or the end point of the spline curve shall meet the following conditions:

$$
\begin{aligned}
& T_{1}=D^{1} \boldsymbol{r}\left(u_{i}\right)-\boldsymbol{Q}_{i}=0 \\
& T_{2}=D^{2} \boldsymbol{r}\left(u_{i}\right)-\boldsymbol{K}_{i}=0
\end{aligned}
$$

where $i=0$ or $i=\mathrm{N}$. If $i=0$ it represents the starting point and if $i=\mathrm{n}$ it represents the end point.

(3) Area Constraint Condition. The area $S$ under the spline curves should meet the condition of the fixed value; that is,

$$
S=S_{0}
$$

where $S_{0}$ is a given area.

At the same time, if necessary, other conditions can be taken into account in the same way mentioned above to complete the corresponding constraints. Finally, the constrained optimization problem can be transformed into the extreme value problem of the unconstrained objective function $I$ by the linear superposition of these constraints:

$$
\begin{aligned}
& I=L_{m}+\lambda A^{\prime}+\mu_{1} T_{1}+\mu_{2} T_{2}+\nu F, \\
& \text { where } A^{\prime}=A+d^{2}
\end{aligned}
$$

where $\lambda, \mu_{1}, \mu_{2}$, and $v$ are all Lagrange operators and $d^{2}$ is a slack variable.

2.2. Hull Deformation Method by Mapping. This section shows how to realize the deformation of the transverse section lines by the fairing B-spline parametric curves. This method is also applicable to other lines of the hull. The hull geometry model is stored in the form of offsets; that is, the hull is discretized into a finite number of transverse section lines. On the $\mathrm{YZ}$ plane, the mapping formula of the coordinate change from the point on the B-spline curve to the point on the transverse section line is expressed as

$$
\begin{aligned}
& x_{u}=x+\boldsymbol{r}_{\delta x u}(x) \\
& z_{u}=z+\boldsymbol{r}_{\delta z u}(z)
\end{aligned}
$$

where $\delta y_{u}$ and $\delta z_{u}$ are the maximum displacement variations of fairing $\mathrm{B}$-spline curve in the $\mathrm{Y}$ axis and $\mathrm{Z}$ axis, respectively; 
$(y, z)$ and $\left(y_{u}, z_{u}\right)$ are the coordinates of any observation point before and after the hull deformation, respectively; $\boldsymbol{r}_{\delta y u}(y)$ and $\boldsymbol{r}_{\delta z w}(z)$ represent the displacement variation of the point on the fairing B-spline curve with the same original coordinates as the observation point when the input parameters are $\delta y_{u}$ and $\delta z$.

Thus, the displacement variations of all points on the Bspline curve can be mapped to the ship hull curve with the same original coordinates. For the B-spline parametric curve itself is highly fairing, while being mapped to the hull, the hull geometry deformation must also be fairing.

In the process of hull form transformation one or more of the fairing B-spline curves can be applied as deformation weights to avoid the geometric distortion. Similarly, assuming that $\delta y_{w}$ and $\delta z_{w}$ are the maximum variations of the curves in the $\mathrm{Y}$ direction and the $\mathrm{Z}$ direction, since they are used as weight functions, $\delta y_{w}$ and $\delta z_{w}$ should meet $0<\delta y_{w} \leq 1$ and $0<\delta z_{w} \leq 1$, respectively. Thus, the coordinate displacement formula of the points on each transverse section line can be expressed as

$$
\begin{aligned}
& x_{u}=x+\boldsymbol{r}_{\delta x u}(x) \cdot \boldsymbol{r}_{\delta x w}(x) \\
& z_{u}=z+\boldsymbol{r}_{\delta z u}(z) \cdot \boldsymbol{r}_{\delta z w}(z)
\end{aligned}
$$

where $\boldsymbol{r}_{\delta y w}(y)$ and $\boldsymbol{r}_{\delta z w}(z)$ represent the weight value of the displacement variation of the point on the fairing B-spline curve with the same original coordinates as the corresponding point on the hull surface when the input parameters are $\delta y_{w}$ and $\delta z_{w}$.

\section{Resistance Prediction Method}

3.1. Total Resistance Calculation Formula. In this article, the hull form transformation is realized by the fairing B-spline parametric curve transformation. The deformation of $\mathrm{B}$ spline parametric curves is realized by adjusting the input characteristic parameters, and then the deformation of the characteristic parameter curve is mapped to the geometric deformation of the hull.

The total resistance can be calculated by the empirical formula as follows:

$$
C_{t}=C_{w}+(1+k) C_{f 0}
$$

where $C_{t}$ is the total resistance coefficient and $C_{w}$ is the wavemaking resistance coefficient which can be obtained by the Rankine source method. $k$ is form factor. $C_{f 0}$ is the frictional resistance coefficient, which can be calculated by the ITTC 1957 model-ship correlation line formula:

$$
C_{f 0}=\frac{0.075}{\left(\lg R_{e}-2\right)^{2}}
$$

where $R e$ is the Reynolds number and $R e=V L / \nu, V$ is the ship speed, $L$ is the length of the waterline, and $v$ is the coefficient of the kinematic viscosity of the fluid.

3.2. Rankine Source Method. Taking the o-xyz as the Cartesian coordinate system fixed on the ship, the $\mathrm{X}$-axis is in the direction and the $\mathrm{Z}$-axis is perpendicular to the surface of the water. The Y-axis is determined by the right hand rule. Assuming that the ship speed is $U$, because of the motion of the hull, the velocity potential $\Phi$ and perturbation potential $\varphi$ with the free surface effect constitute the total velocity potential $\phi$ of flow field around the ship, whose expression is $\phi=\Phi+\varphi$. In the potential flow, the ideal fluid is nonviscous, incompressible, and nonrotating. Then in the fluid computational domain the total velocity potential $\varphi$ subjects to the Laplace equation and the following boundary conditions:

$$
\nabla^{2}(\Phi+\varphi)=0
$$

(1) The Hull Boundary Condition. This is

$$
\nabla(\Phi+\varphi) \cdot \vec{n}=0
$$

where $\vec{n}$ is the unit outward normal vector of the hull surface and is represented by $\vec{n}=(n x, n y$, and $n z)$.

\section{(2) The Free Surface Condition. This is}

$$
\frac{1}{2} \phi_{x}(\nabla \phi \cdot \nabla \phi)_{x}+\frac{1}{2} \phi_{y}(\nabla \phi \cdot \nabla \phi)_{y}+g \phi_{z}=0
$$

where the subscripts denote the partial derivatives and $g$ is the gravitational acceleration.

\section{(3) The Radiation Condition. This is}

$$
\begin{aligned}
\nabla \varphi & \longrightarrow(U, 0,0) \\
\text { as } x^{2}+y^{2}+z^{2} & \longrightarrow \infty
\end{aligned}
$$

Employ the Rankine source panel method by the iteration procedure to solve the above boundary conditions. Once the velocity potential $\phi$ is resolved, the free surface wave elevation $\eta$ can be obtained. In addition, through the instrumentality of the Bernoulli equation, the pressure coefficient $C p$ at each panel can be defined as

$$
C_{p}=1-\frac{\nabla \phi \cdot \nabla \phi}{U^{2}}-2 \frac{z}{F n^{2}}
$$

where $F_{n}$ is the Froude number with the expression, $F_{n}=$ $U / \sqrt{g L_{W L}}$. Hence the wave-making resistance coefficient $C_{w}$ can be solved by integral calculus of the pressure throughout the wetted hull surface $S$ and formulated as

$$
C_{w}=-\frac{1}{S} \int_{S} C_{p} n_{x} d s
$$

When each resistance coefficient component is obtained, the total calm water resistance $R_{t}$ could then be determined by $R_{t}$ $=0.5 \rho U^{2} S C_{t}$, where $\rho$ is the density of fluid.

3.3. Validation of the Total Resistance Calculation Method for KCS. KCS (KRISO Container Ship) is a modern container ship designed by Korea Research Institute of Ships and Ocean Engineering. It has been selected as the standard form of 


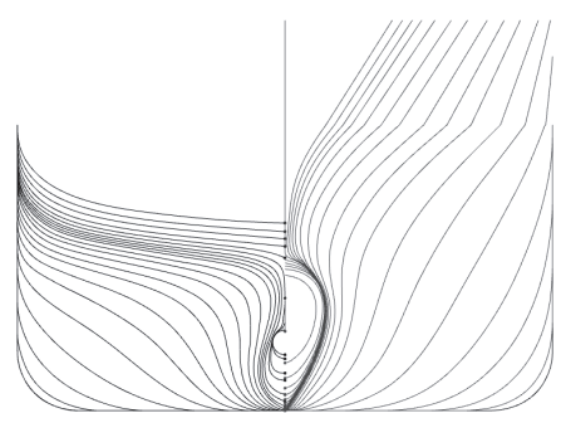

Figure 1: The body plan of the KCS.

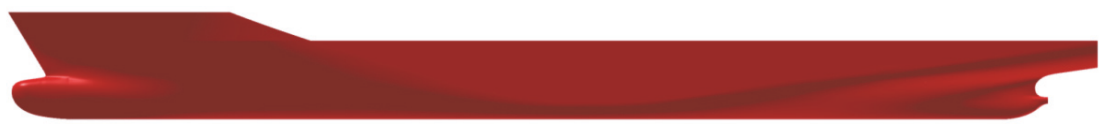

Figure 2: The side view of the KCS.

TABLE 1: Principal characteristics of the KCS model.

\begin{tabular}{lccc}
\hline Principal dimensions & Symbol & Unit & Value \\
\hline Scale & $\lambda$ & {$[-]$} & 31.5994 \\
Length between & $L_{P P}$ & {$[\mathrm{~m}]$} & 7.2786 \\
perpendiculars & $L_{W L}$ & {$[\mathrm{~m}]$} & 7.3568 \\
Waterline length & $B$ & {$[\mathrm{~m}]$} & 1.0190 \\
Beam & $D$ & {$[\mathrm{~m}]$} & 0.5696 \\
Moulded depth & $T$ & {$[\mathrm{~m}]$} & 0.3418 \\
Draught & $\nabla$ & {$\left[\mathrm{m}^{3}\right]$} & 1.6493 \\
Displacement & $\mathrm{S}$ & {$\left[\mathrm{m}^{2}\right]$} & 9.5579 \\
Wetted surface area & $C_{B}$ & {$[-]$} & 0.6508 \\
Block coefficient & $C_{M}$ & {$[-]$} & 0.9849 \\
Midship section coefficient & $C_{P}$ & {$[-]$} & 0.6608 \\
Prismatic coefficient & $F n$ & {$[-]$} & 0.26 \\
Design speed & &
\end{tabular}

CFD results validation by Goteborg ship CFD symposium and Tokyo ship CFD symposium, with a wealth of open test data and a large number of numerical results. The principal dimensions are listed in Table 1 . The body plan, the side view, and the mesh generation are shown in Figures 1, 2, and 3, respectively. There are 1132 hull surface meshes and 2485 free surface meshes.

According to the calculation method of the ship total resistance mentioned above, five speed points are selected for the hydrodynamic resistance performance evaluation and the corresponding calculation conditions are consistent with the ship model test data published in the ship hydrodynamic CFD symposium. Comparison of the total resistance coefficient between the calculated results and the open test results is shown in Figure 4. It can be seen from the figure that the numerical results are in good agreement with the open test results. The overall error is controlled within $2 \%$, which confirms the validity of the calculation method.
Comparisons of wave profile and longitudinal wave cuts profiles between the calculation results and test results are shown in Figures 5 and 6, respectively, which also show that the calculated results are in good agreement with the experimental results. The wave pattern calculated is shown in Figure 7.

The comparative analysis shows that the empirical formula combined with the Rankine source method based on the potential flow theory to predict the resistance performance of ship has a high reliability, which can meet the requirements of ship hull optimization well.

\section{Optimization Strategy}

The optimization strategy is a critical part of the resistance optimization design methodology for bow and stern line. A large number of studies related to the optimization strategies have been explored and implemented for the ship design. In this study, the NSGA-II (Nondominated Sorting Genetic Algorithm II) is used as the resistance optimization algorithm for searching for the optimal hull form with a minimal ship total resistance considering all speed range.

\subsection{Fast Elitist Nondominated Sorting Genetic Algorithm}

(1) The Fast Nondominated Sorting Method. The fast nondominated sorting method is implemented. Each individual $i$ is set with two attributes $S_{\mathrm{i}}$ and $n_{\mathrm{i}}$. The former is the set of solution individuals dominated by the individual $i$; the latter is the number of solution individuals that dominate the individual $i$. At first, all individuals in the population are found in terms of $n_{\mathrm{i}}=0$ and put into the current set $F_{1}$. Then, for each individual $j$ in the current set $F_{1}$, every individuals set $S_{j}$ dominated by individual $j$ is examined. The $n_{\mathrm{k}}$ of each individual $k$ belonging to individuals set $S_{\mathrm{j}}$ is decremented by one. If $n_{\mathrm{k}}-1=0$, then the individual $k$ is the nondominant individual within $S_{\mathrm{j}}$ and is put into another set $H$. Finally, the same nondominated rank $\mathrm{i}_{\text {rank }}$ is assigned to 


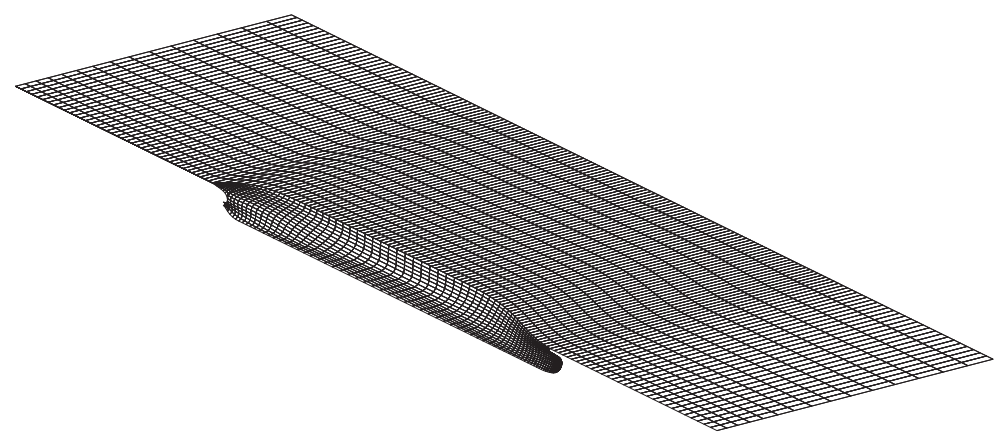

Figure 3: The mesh generation of the hull and free surface.

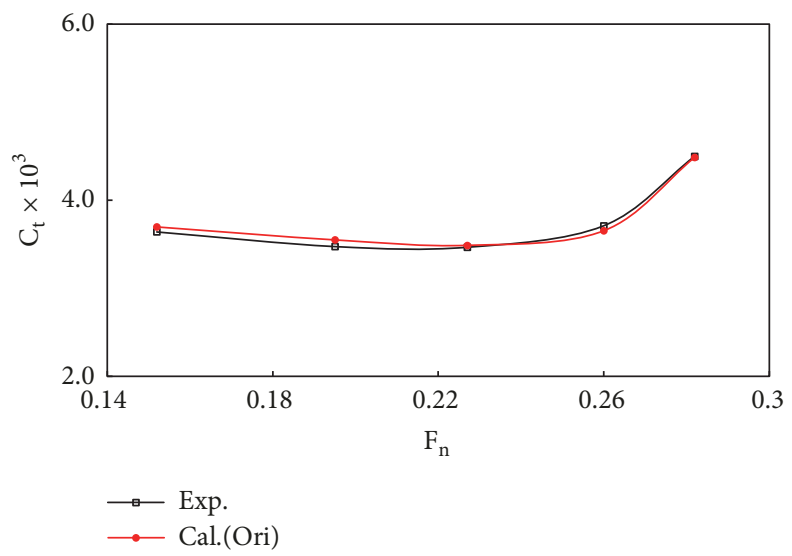

FIGURE 4: Comparison of total resistance of the KCS between experiment and calculation.

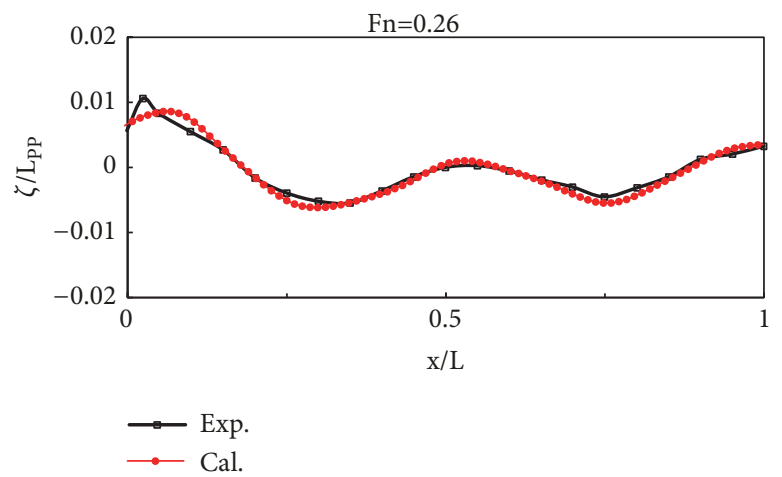

FIGURE 5: Comparison of wave profile of the KCS between experiment and calculation at $F n=0.26$.

all the individuals within $F_{1}$ and the same process continues until all individuals are graded.

(2) Density Estimation and the Crowded Comparison Operator. In order to ensure the diversity of the population in the process of optimization calculation, the technology of density estimation is employed, representing the density of any individual $i$ as crowding distance $i_{\mathrm{d}}$, that is, the smallest cube containing only the individual $i$. When $i_{\mathrm{d}}$ is smaller, it means that the feasible solutions are concentrated around an individual $i$. For maintaining the diversity of the population, an operator for density estimation and the crowded comparison is needed to ensure that the algorithm can converge to a uniform distribution of Pareto-optimal front. After sorting and crowded comparison, any individual $i$ in the population has two attributes, nondomination rank $i_{\text {rank }}$ and crowding distance $i_{d}$. For any two individuals $i, j$, if the conditions $i_{\text {rank }}<j_{\text {rank }}$ or $i_{\text {rank }}=j_{\text {rank }}$ but $i_{d}<j_{d}$ meet, then the individual $i$ is preferred.

(3) Algorithm Procedure. In this study, the step-by-step procedure shows that NSGA-II algorithm is simple and straightforward. A random parent population $P_{0}$ is created initially. Then a combined population $R_{t}=P_{t} \cup Q_{t}$ is formed in which the $t$ th is the generation of the proposed algorithm; therefore the population $R_{t}$ will be of size $2 N$. The population 


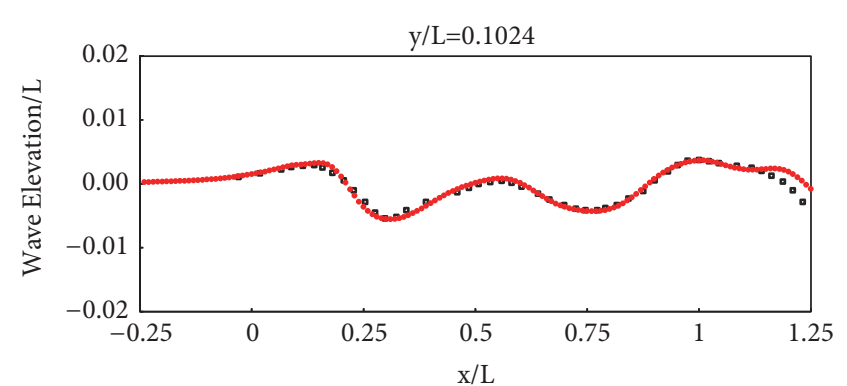

- Exp.

- Cal.

(a)

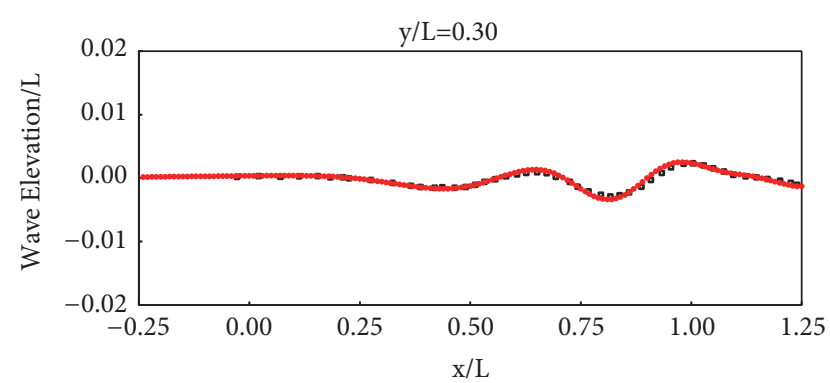

- Exp.

- Cal.

(c)

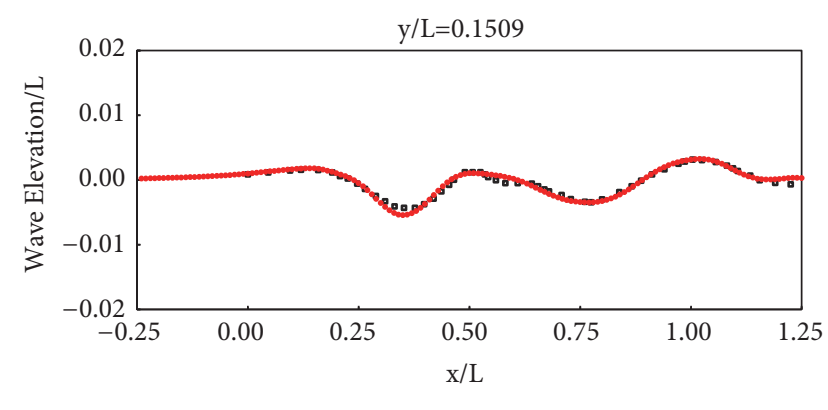

- Exp.

- Cal.

(b)

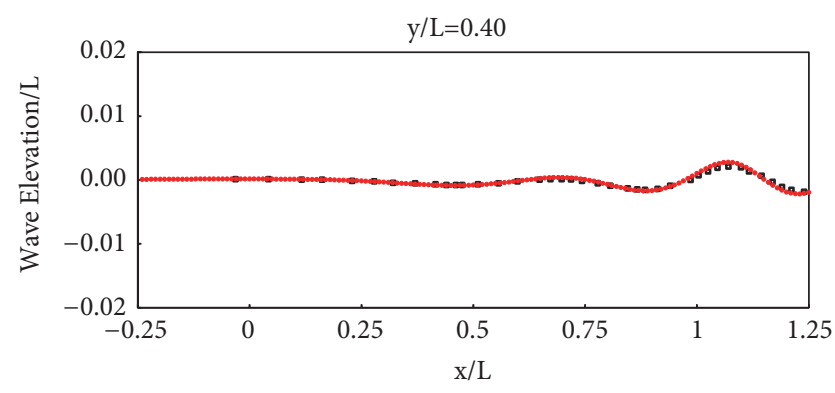

- Exp.

- Cal.

FIGURE 6: Longitudinal wave cuts profiles of the Series 60 ship at different values of $y / L$ under the condition of $F n=0.26:(a)$ y/L $=0.1024 ;(b)$ $y / L=0.1509 ;(c) y / L=0.3 ;(d) y / L=0.4$.

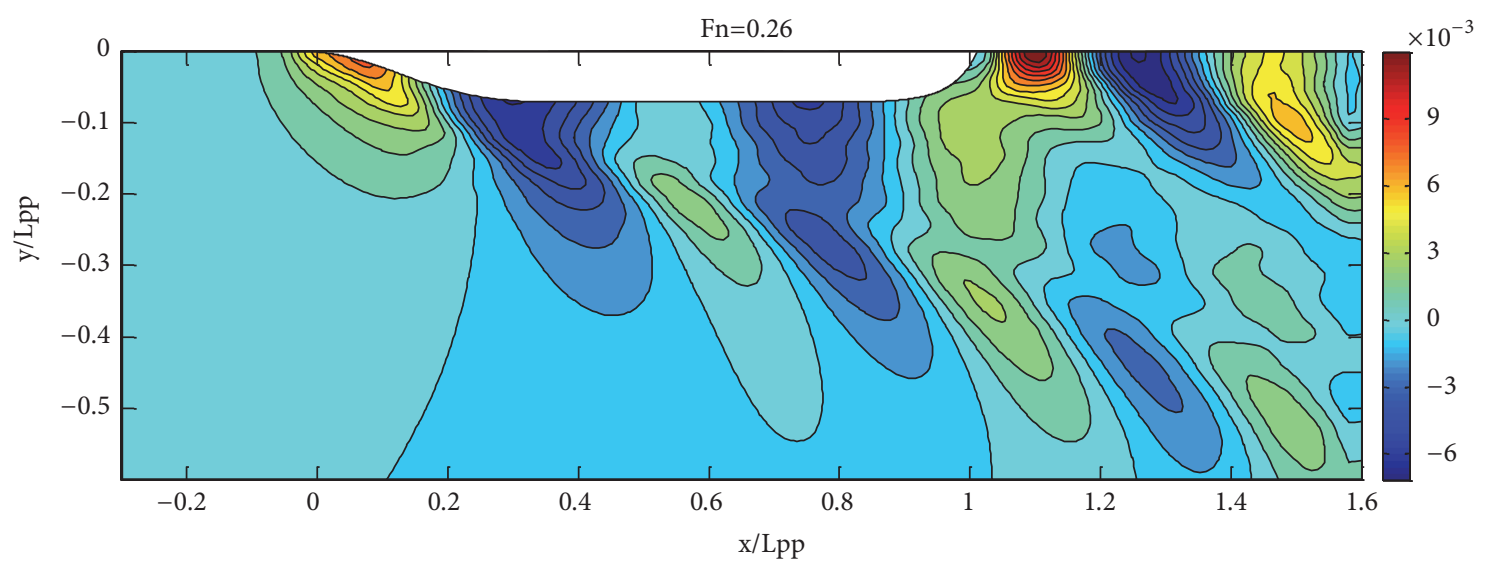

FIGURE 7: Wave pattern of the KCS at $F n=0.26$.

$R_{t}$ is sorted according to nondomination. The new parent population $P_{t+1}$ is formed by adding solutions from the first front till the size exceeds $N$. Thereafter, the solutions of the last accepted front are sorted according to $\geqslant n$ and the first $N$ points are picked. This population of size $N$ is now used for selection, crossover, and mutation to create a new population $Q_{t+1}$ of size $N$. It is noted that we use a binary tournament selection operator but the selection criterion is now based on the niched comparison operator $\geqslant n$. The NSGA-II algorithm flowchart is shown in Figure 8.

4.2. Optimization Design Flow of Hull Lines. The implementation of the hydrodynamic optimization system required the use of three main components presented above, namely, the hull form transformation, the resistance prediction by Rankine source method, and the optimization strategy. Through 


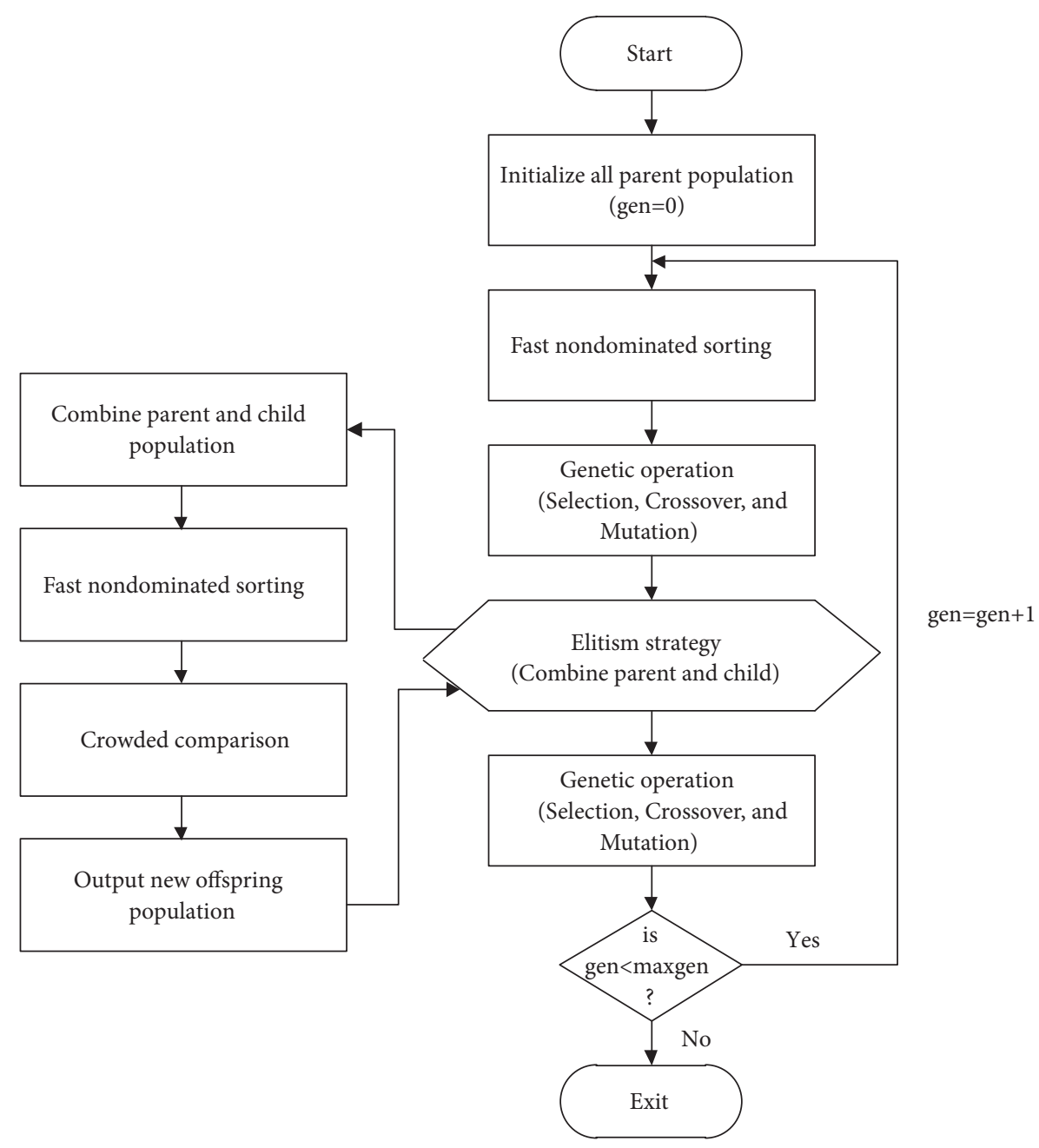

FIGURE 8: The NSGA-II algorithm flowchart.

the analysis and establishment of hull form optimization model proposed above, the optimization design process of hull lines is shown in Figure 9.

\section{KCS Ship Optimization}

\subsection{Optimization Based on Single Design Speed}

(1) Definition of Optimization Problem for Single Design Speed

(a) Objective Function. The total resistance at the design speed is used as the optimization goal:

$$
F=\frac{C_{t}}{C_{t 0}}
$$

where $C_{t 0}$ is the total resistance coefficient of original hull and $C_{t}$ is the total resistance coefficient of the feasible solution in the optimization process.

(b) Design Variables. Because the KCS ship has a larger bulbous bow as well as a complex shape of stern surface, when it is sailing, the first shear wave and the tail wave will interfere with each other after the ship. The interference may be beneficial or harmful to wave making resistance. Therefore, the bow and the stern are selected as the optimization design area, as shown in Figure 10.

A total of 7 variables are set for the bow area, that is $d x_{1}, d y_{1}, d y_{2}, d y_{3}, d y_{4}, d z_{1}$, and $d z_{2}$ which are mainly used to control the expansion changes of bulbous bow in the direction of $\mathrm{X}$ axis, the harmomegathus changes in the direction of $\mathrm{Y}$ axis, the translational changes of the bow in the direction of $\mathrm{Z}$ axis, and the precursor smooth transition between bulbous bow and hull. A total of 3 variables are set for the stern area, which are $d z_{3}, d z_{4}$, and $d z_{5}$ mainly used to control the translational changes of the stern in the direction of $\mathrm{Z}$ axis, the sag at the upper end of the stern, and the uplift of the lower end of the stern. The different types of transformation for the KCS ship in offsets circumvented with the above 7 variables are shown in Figures 11-17, where original hull lines are marked with red and the transformed hull lines are marked with blue.

(c) Constraints Conditions. The constraint of the change of displacement is $|\nabla / \nabla 0-1|<1 \%$; the constraint of the change of wetted surface is $\left|\mathrm{S} / \mathrm{S}_{0}-1\right|<1 \%$. The constraint conditions 


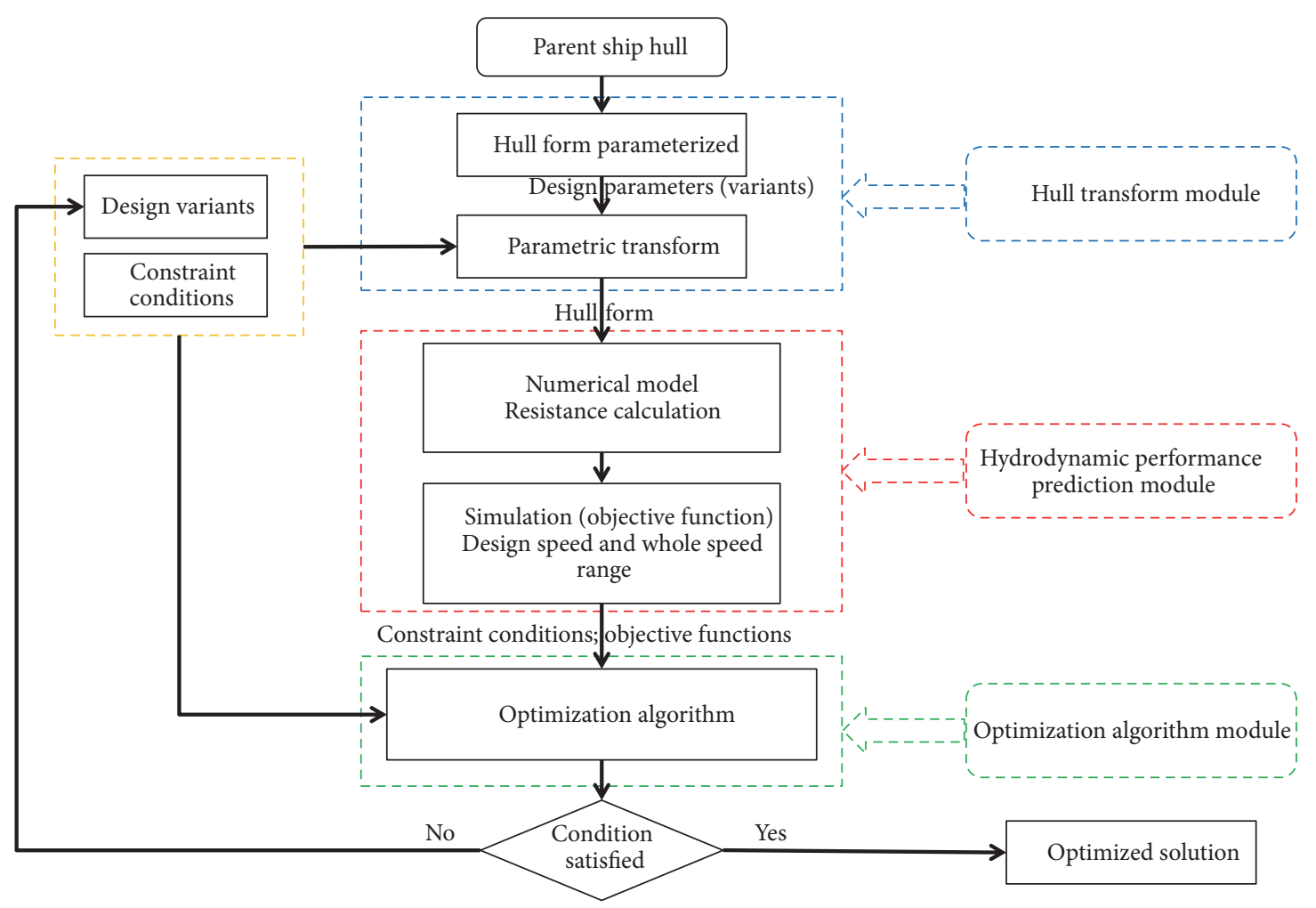

FIGURE 9: The process chart of hull lines optimization.

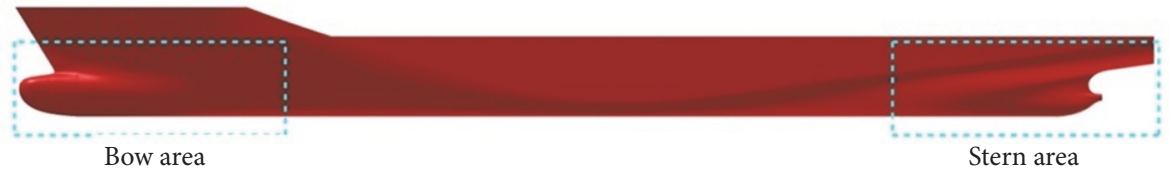

FIGURE 10: The transformation regions of the KCS forebody and aftbody, respectively.

on the 10 design variables that control the translation of the hull are listed as follows:

$$
\begin{aligned}
& -2.0 \leq d x_{1} \leq 2.0 \\
& -2.5 \leq d y_{1} \leq 1.5 \\
& 160 \leq d y_{2}\left(^{\circ}\right) \leq 180 \\
& -0.2 \leq d y_{3} \leq 1.2 \\
& -1.0 \leq d y_{4} \leq 0.2 \\
& -1.5 \leq d z_{1} \leq 1.5 \\
& -0.5 \leq d z_{2} \leq 2.5 \\
& -0.3 \leq d z_{3} \leq 0.6 \\
& -0.6 \leq d z_{4} \leq 0.3 \\
& -0.3 \leq d z_{5} \leq 0.8
\end{aligned}
$$

(2) Optimization Results for Single Design Speed. After setting the population size $\mathrm{M}=20$, the evolution generations $\mathrm{N}=20$,
TABLE 2: The specific presentations of OptS01.

\begin{tabular}{lccccc}
\hline & & $d x_{1}$ & 1.6 & $d z_{1}$ & 0.8 \\
$F$ & $92.47 \%$ & $d y_{1}$ & -2.0 & $d z_{2}$ & -0.4 \\
$\left|\nabla / \nabla_{0}-1\right|$ & $0.74 \%$ & $d y_{2}$ & 174 & $d z_{3}$ & 0.15 \\
$\left|S / S_{0}-1\right|$ & $0.93 \%$ & $d y_{3}$ & 0.01 & $d z_{4}$ & -0.48 \\
& & $d y_{4}$ & -0.7 & $d z_{5}$ & 0.47 \\
\hline
\end{tabular}

the crossover probability $\mathrm{Pc}=0.8$, and the mutation probability $\mathrm{Pm}=0.3$, the optimization based on the design speed is carried out. The convergence courses for the objective function and various design variables are shown in Figures 18 and 19 , respectively.

As shown in Figures 18 and 19, the objective function and design variables are convergent in the last iteration. The final convergence solution is marked by the red boundary line, denoted by OptS01. The design variables and constraints of the optimal one are listed in Table 2. Comparison of the transverse section lines between the optimal hull and original 


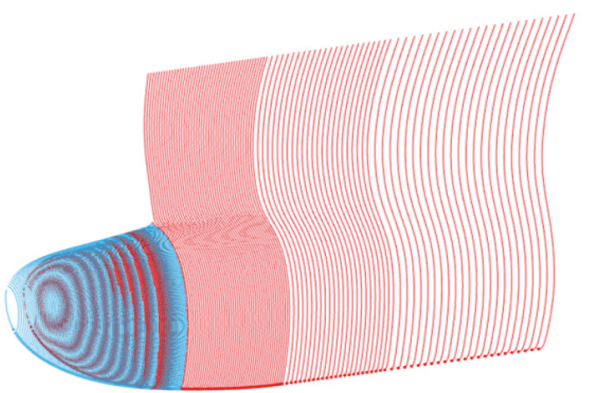

FIGURE 11: X-direction transformations of forebody offsets compared to original hull form.

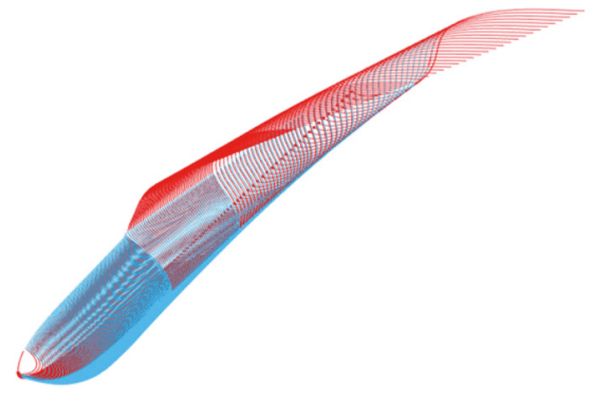

FIGURE 12: Y-direction transformations of forebody offsets compared to original hull form.

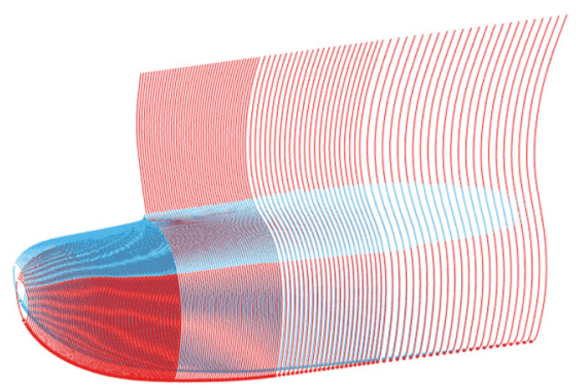

FIGURE 13: Z-direction transformations of forebody offsets compared to original hull form.

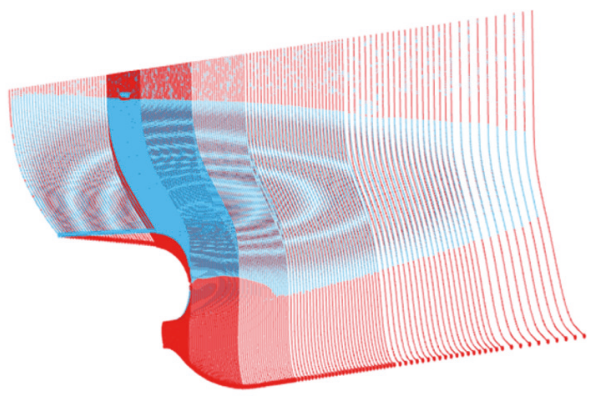

FIGURE 14: Z-direction transformations of aftbody offsets compared to original hull form.

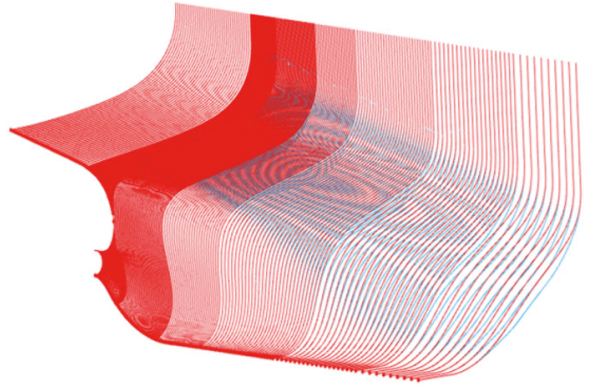

FIGURE 15: Upper concave transformations of aftbody offsets compared to original hull form.

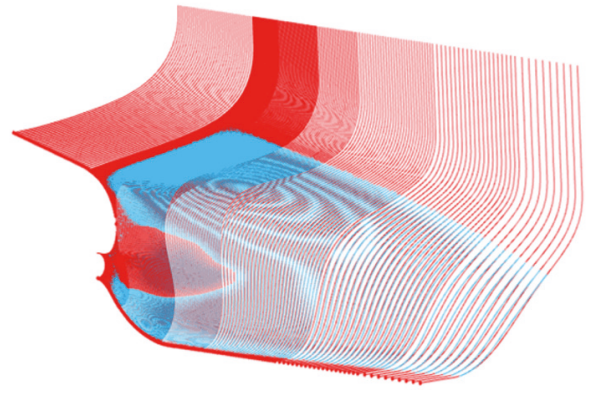

FIGURE 16: Lower convex transformations of aftbody offsets compared to original hull form.

hull is shown in Figure 20 and comparison of surface waves at design speed of $F n=0.26$ is shown in Figure 21.

As shown in Figure 21, the first peak wave moves backward in the vicinity of the bow and the tail peak wave maximum amplitudes of the optimized hull are obviously smaller than those of the original hull.

\subsection{Optimization Based on Whole Speed Range}

(1) Definition of Optimization Problem for Whole Speed Range. In the optimization based on the whole speed range, the design area and the hull deformation are consistent with the previous text. Therefore, the design variables and functional constraints (drainage volume and wet surface area) will remain unchanged. The target condition is changed to 5 


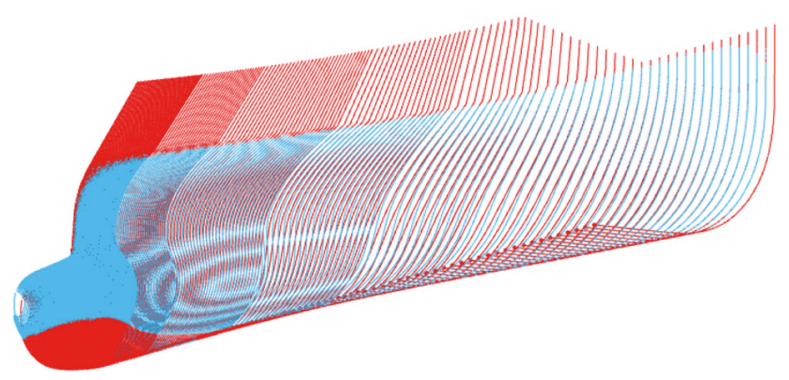

FIGURE 17: Fair transition transformations of forebody offsets compared to original hull form.

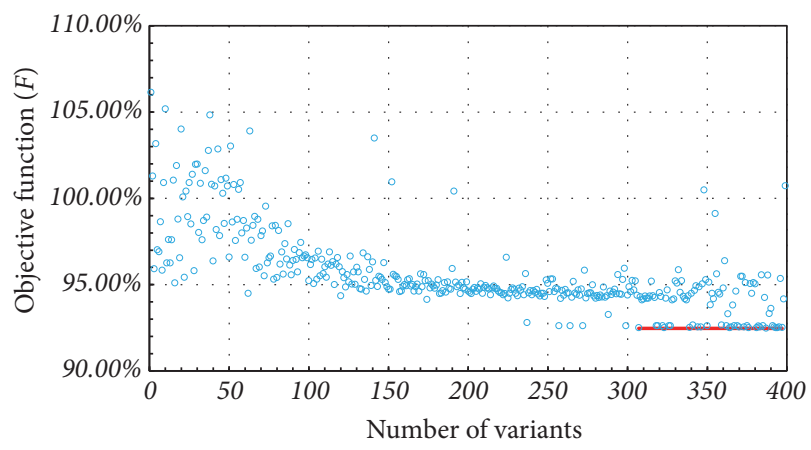

FIGURE 18: The convergence course for the objective function.

typical speed points covering the whole speed range of $F n=$ $0.15 \sim 0.30$, which is the normal operation condition area of the container ship. Therefore, the total resistance coefficients at the 5 speed points are all used as subobjective functions, which are expressed as

$$
\begin{aligned}
& F_{1}=\frac{C_{t 1}}{C_{t 01}} \\
& F_{2}=\frac{C_{t 2}}{C_{t 02}} \\
& F_{3}=\frac{C_{t 3}}{C_{t 03}} \\
& F_{4}=\frac{C_{t 4}}{C_{t 04}} \\
& F_{5}=\frac{C_{t 5}}{C_{t 05}}
\end{aligned}
$$

where, $C_{t 01}, C_{t 02}, C_{t 03}, C_{t 04}$, and $C_{t 05}$ are the total resistance coefficients of KCS, at the speeds of $F n=0.152, F n=0.195$, $F n=0.227, F n=0.26$, and $F n=0.282$, respectively; $C_{t 1}, C_{t 2}$, $C_{t 3}, C_{t 4}$, and $C_{t 5}$ are the total resistance coefficients of the corresponding speed points of feasible scheme in the process of optimization; $F_{4}$ is the subobjective function for design speed of $F n=0.26$.

(2) Optimization Results for Whole Speed Range. After setting the population size $\mathrm{M}=20$, the evolution generations $\mathrm{N}=20$, the crossover probability $\mathrm{Pc}=0.8$, and the mutation probability $\mathrm{Pm}=0.3$, the optimization based on the whole speed range is carried out. The solution set of each subobjective function is shown in Figures 22-25.

For the optimization objective function of KCS at the design speed point is the subobjective function $F_{4}$, only the solution sets of the other subobjective functions relative to the design speed objective function $F_{4}$ are showed in Figures 22-25. Because in the absence of special requirements or restrictions the optimal solution at the design speed point is still the main object, the optimal solution of the multiobjective optimization can be obtained from the "exclusive" Pareto solutions of the subobjective functions relative to the subobjective function $F_{4}$. In Figures 22-25, the feasible solutions are shown together with the infeasible solutions beyond the constraints. In order to select the optimal solution from the solution set conveniently, the feasible solutions will be marked by the blue hollow " $\bigcirc$ " and the infeasible solutions will be marked by the purple hollow " $\Delta$ ". The two optimal solutions Opt01 and Opt02 are obtained from the solution set, which are marked by red solid “ $\square$ ". At the same time, the convergence courses for various design variables of optimization are shown in Figure 26.

From the Pareto solution set in Figures 22-25 it can be seen that there are two final optimal solutions at the speeds of $F n=0.152, F n=0.195, F n=0.227, F n=0.26$, and $F n=0.282$, respectively, denoted by Opt01 and Opt02, whose objective function, corresponding design variables, and constraints are listed in Table 3.

Comparison of the transverse section lines between the optimal schemes Opt01 and Opt02 and the original hull is shown in Figure 27. It can be seen that, compared to the original hull, the bulbous bows of Opt01 and Opt02 


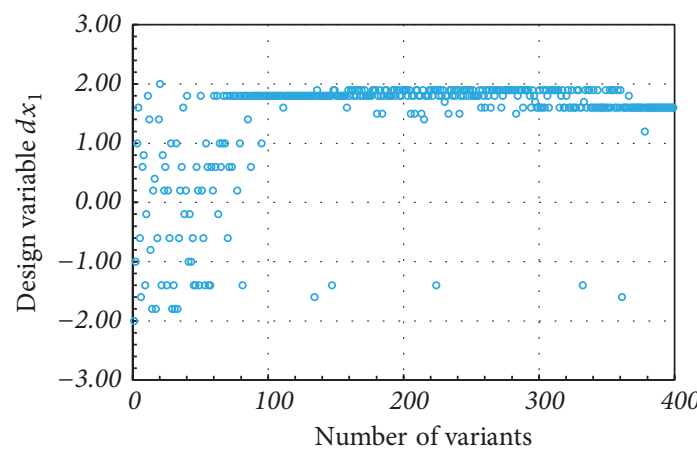

(a)

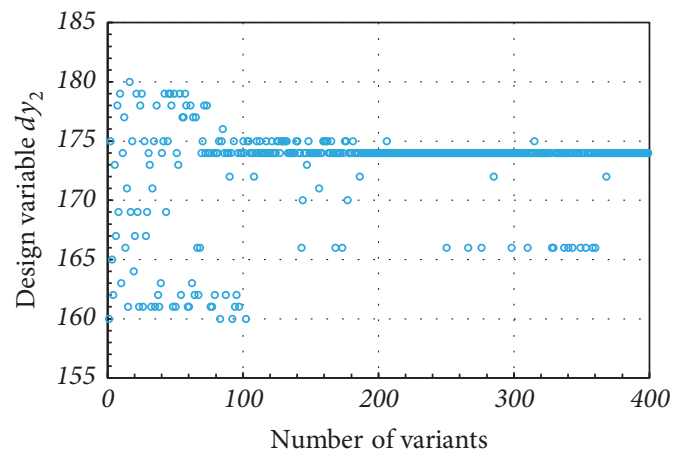

(c)

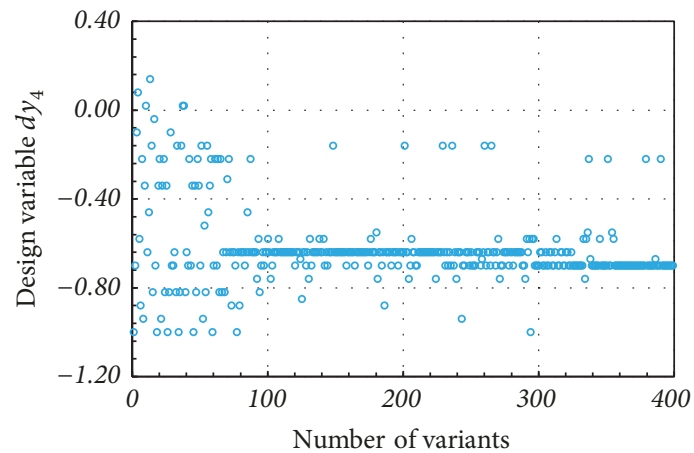

(e)

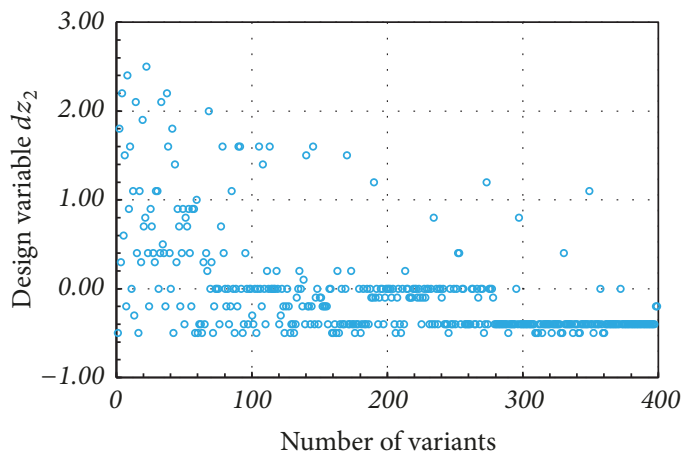

(g)

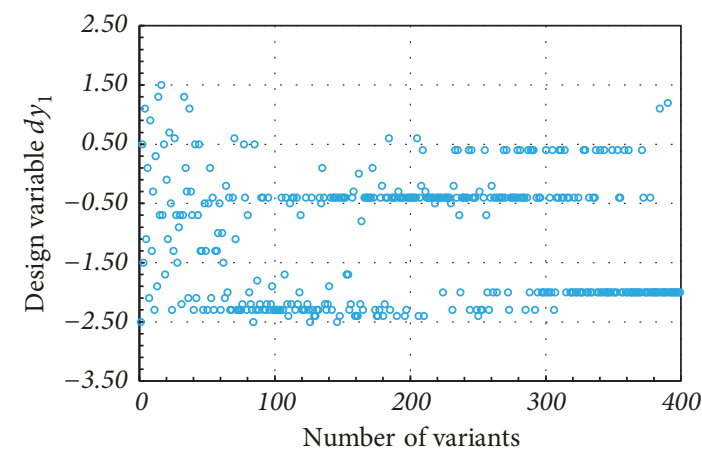

(b)

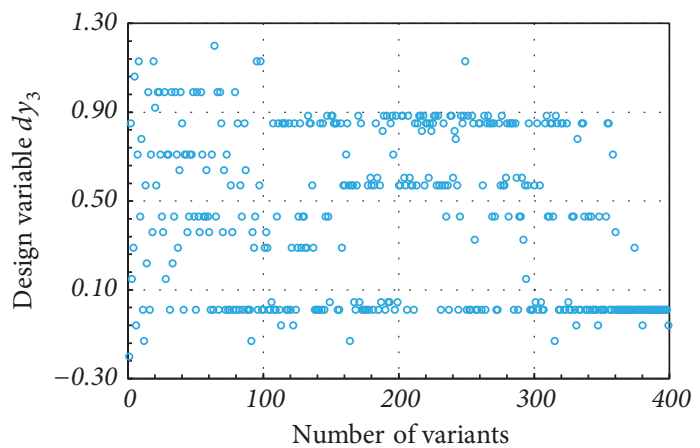

(d)

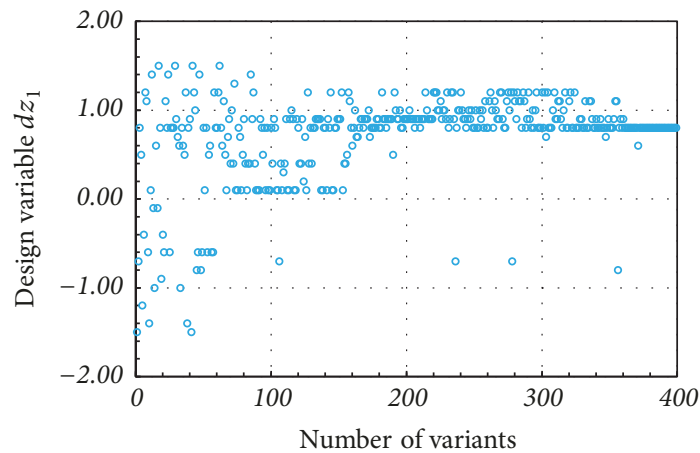

(f)

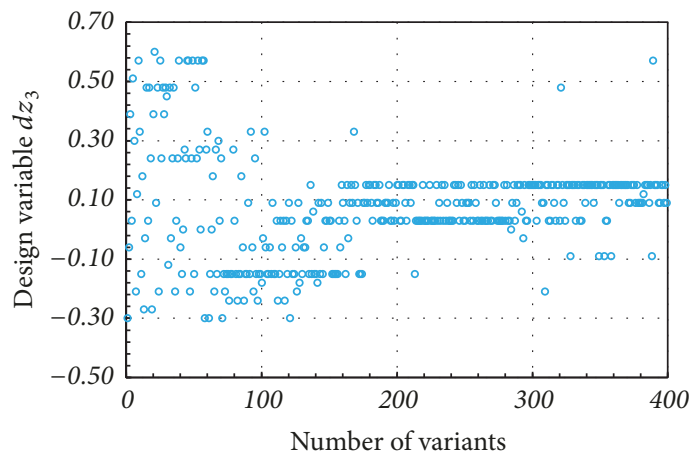

(h)

Figure 19: Continued. 


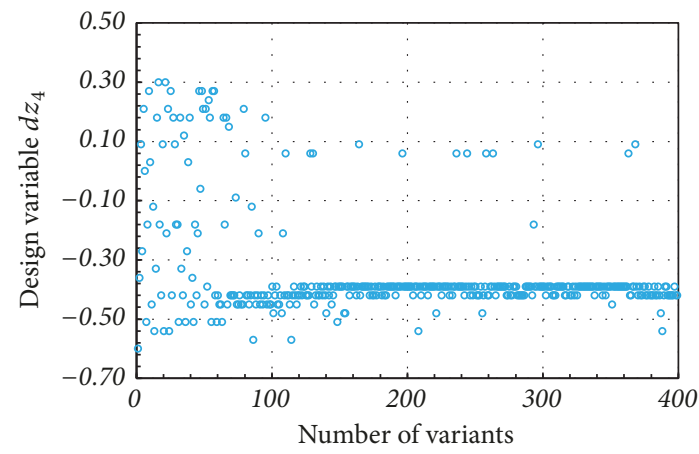

(i)

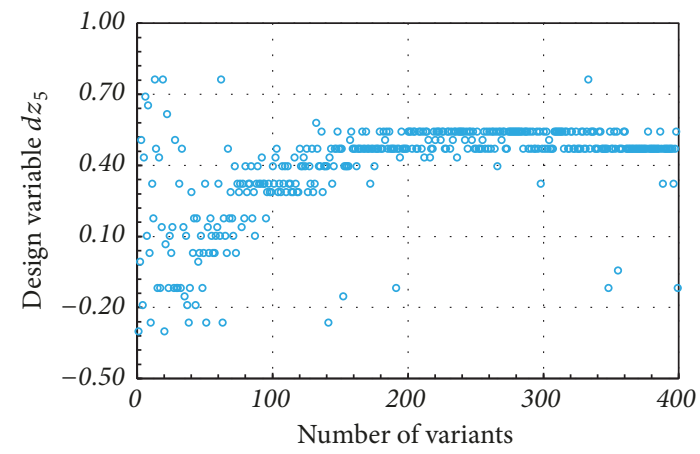

(j)

FIGURE 19: The convergence course of single design speed optimization for various design variables: (a) $d x_{1}$, (b) $d y_{1}$, (c) $d y_{2}$, (d) $d y_{3}$, (e) $d y_{4}$, (f) $d z_{1}$, (g) $d z_{2}$, (h) $d z_{3}$, (i) $d z_{4}$, and (j) $d z_{5}$.

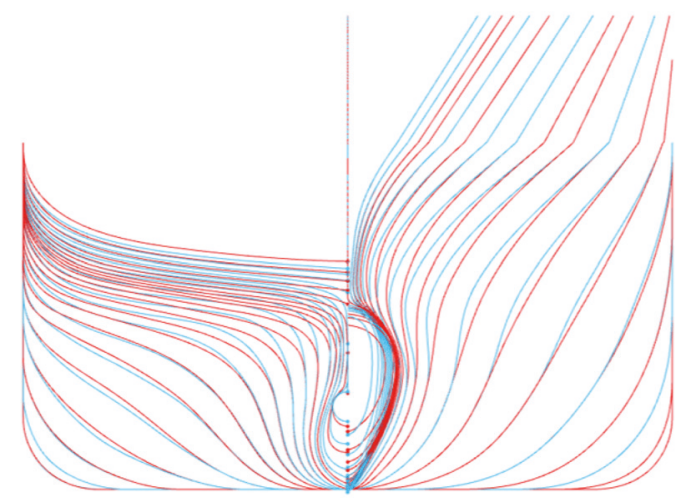

FIGURE 20: Comparison of the optimized hull form (blue) and original hull form (red).

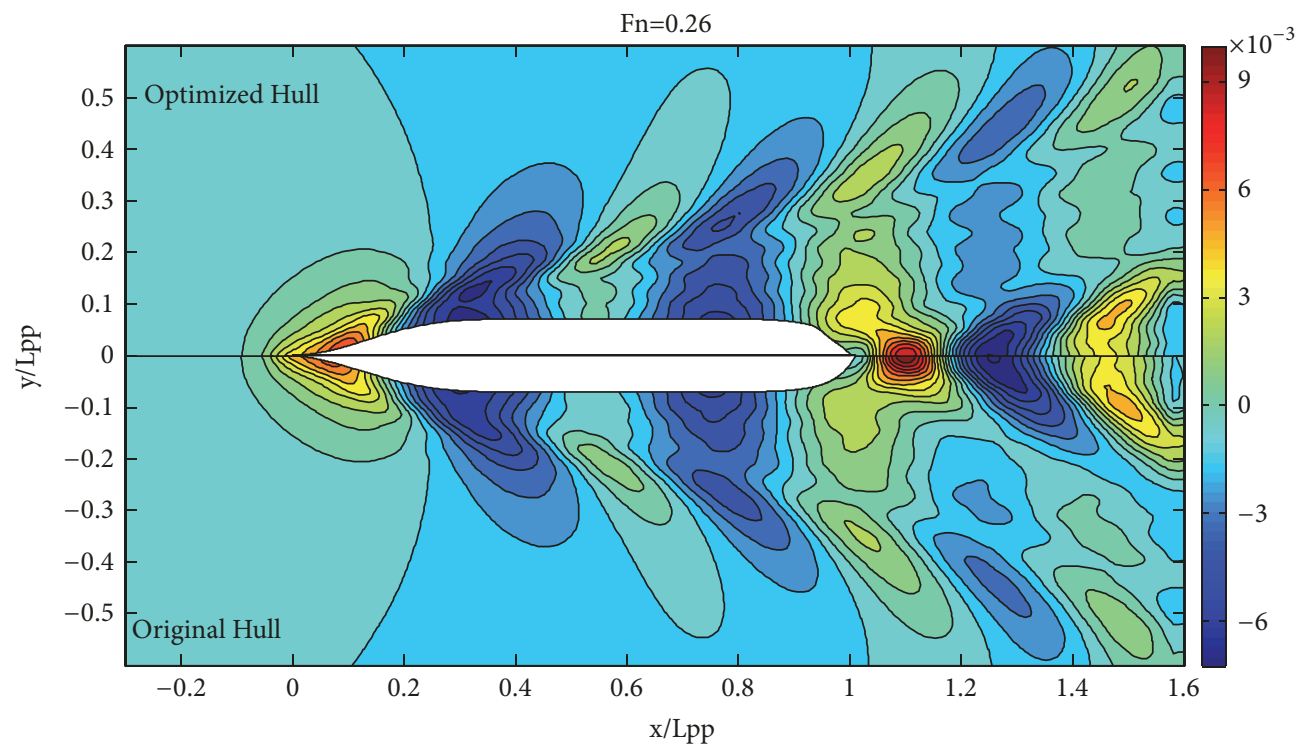

FIGURE 21: Comparison of wave patterns between the optimized hull and original hull at $F n=0.26$. 


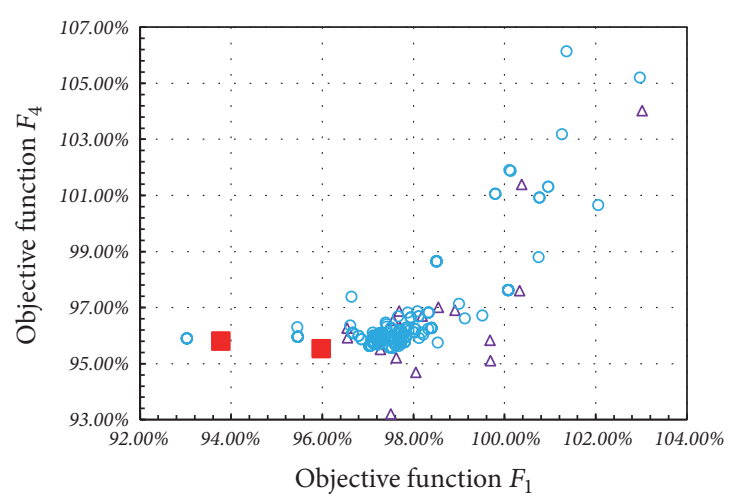

Figure 22: The solution set of objective functions $F_{1}$ and $F_{4}$.

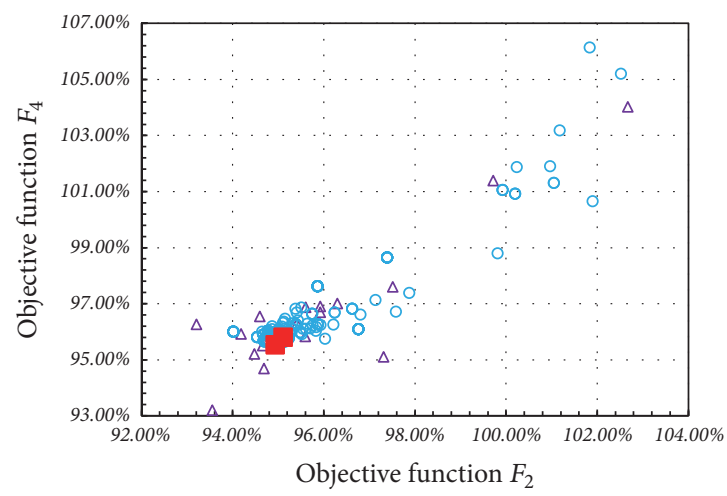

FIgURE 23: The solution set of objective functions $F_{2}$ and $F_{4}$.

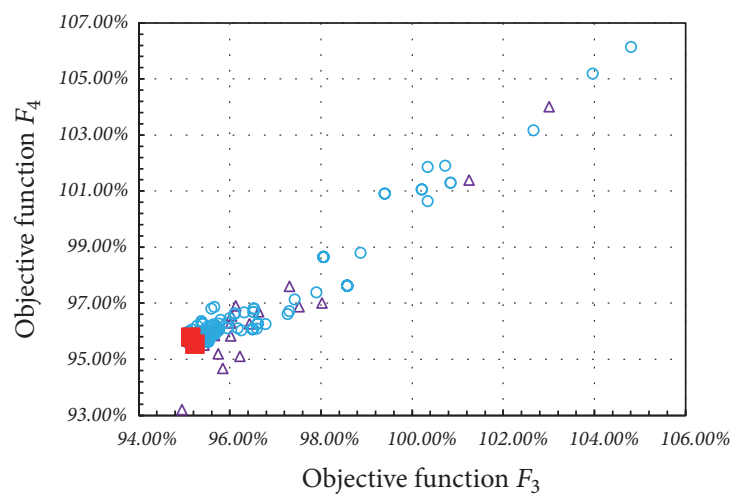

FIgURE 24: The solution set of objective functions $F_{3}$ and $F_{4}$.

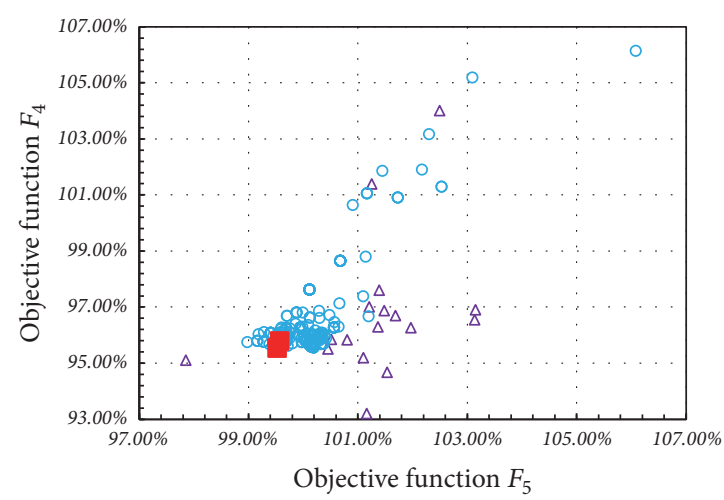

FIgURE 25: The solution set of objective functions $F_{5}$ and $F_{4}$. 


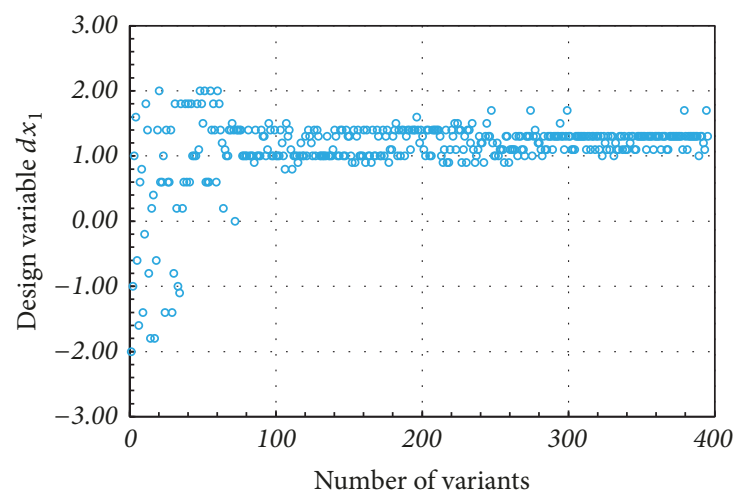

(a)

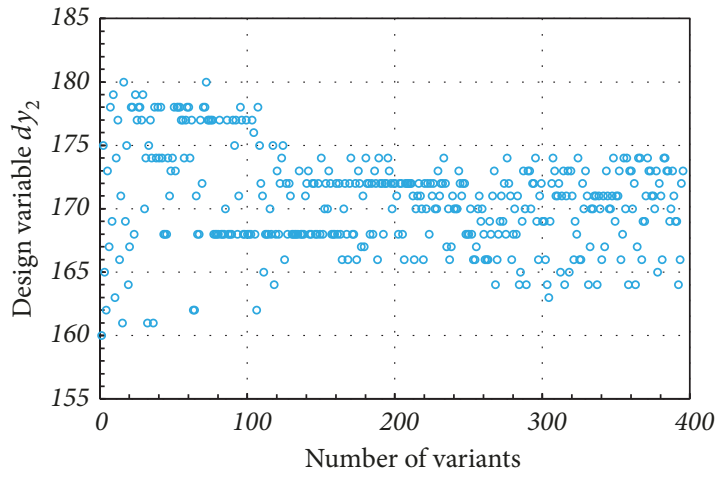

(c)

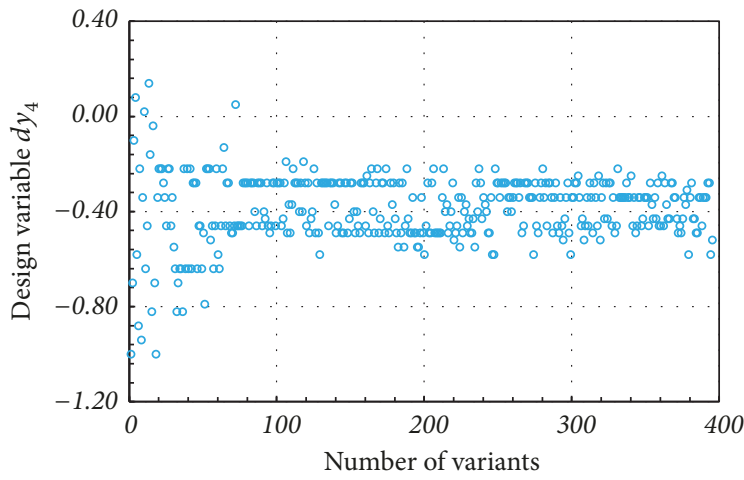

(e)

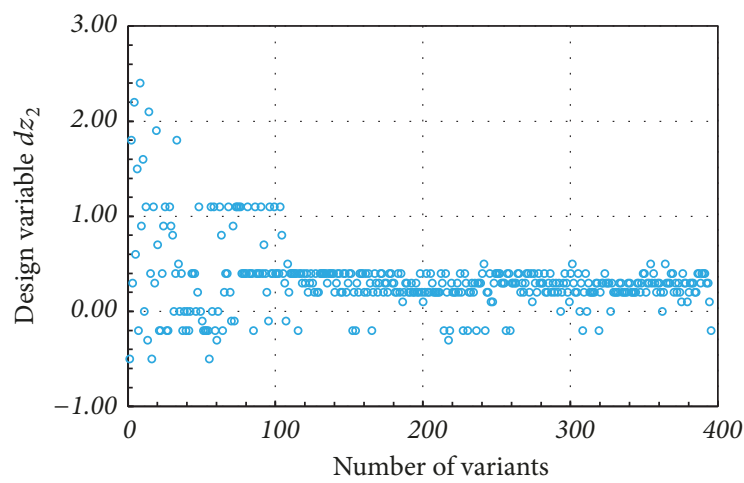

(g)

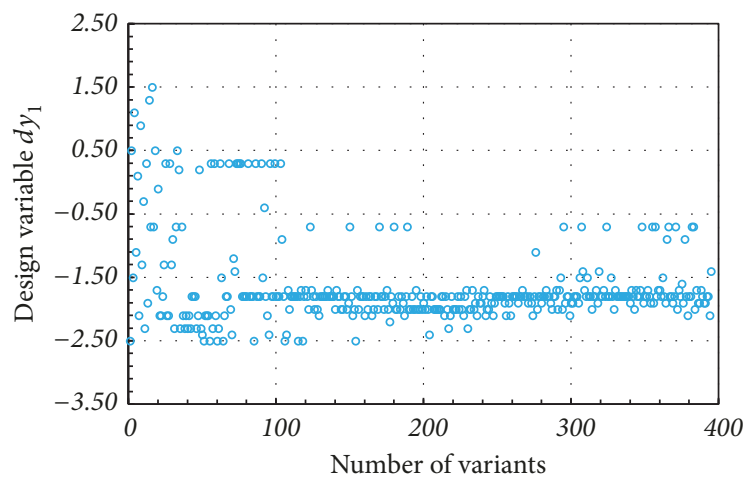

(b)

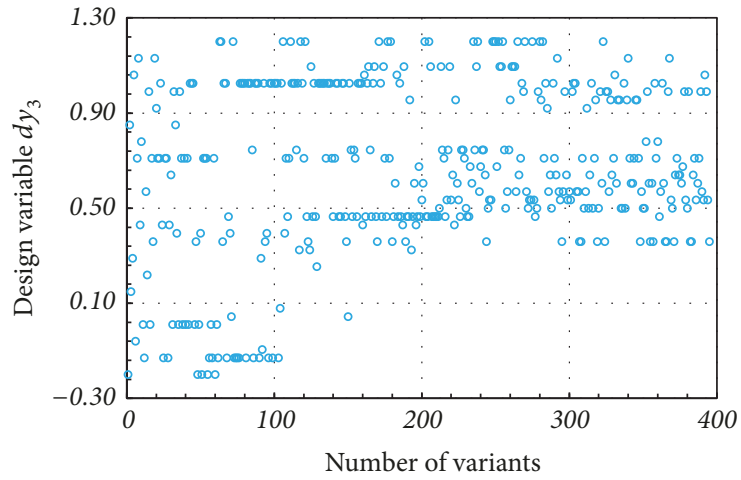

(d)

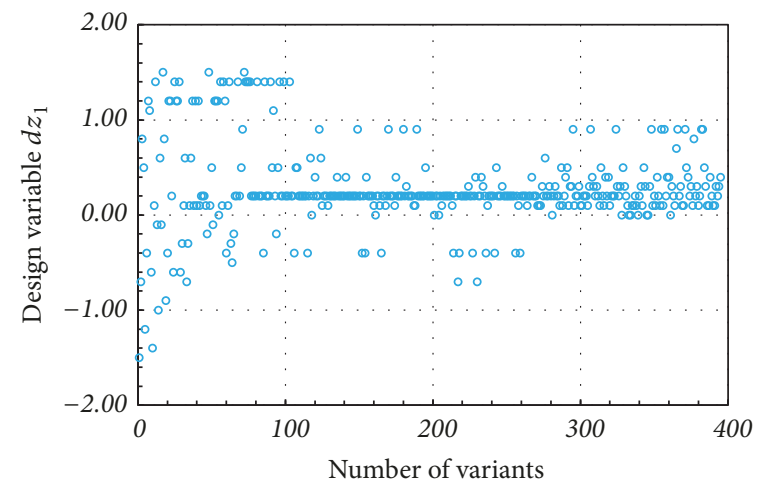

(f)

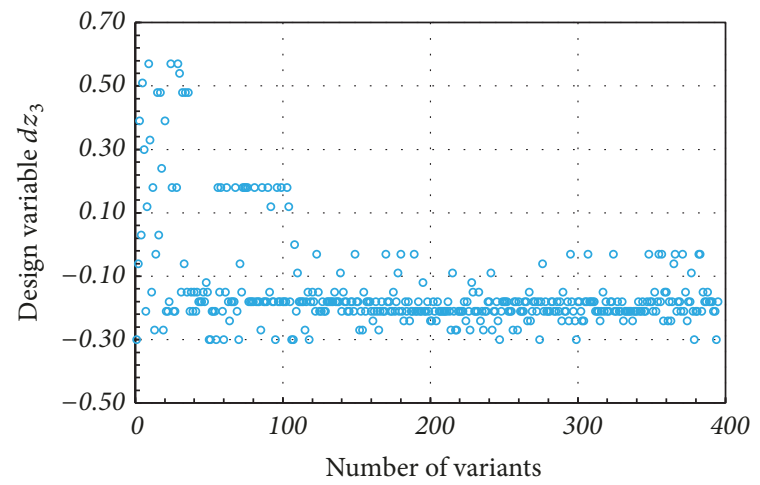

(h)

Figure 26: Continued. 


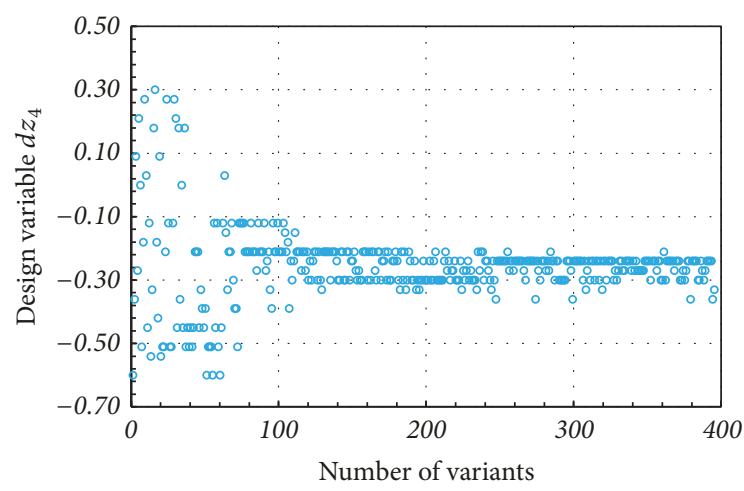

(i)

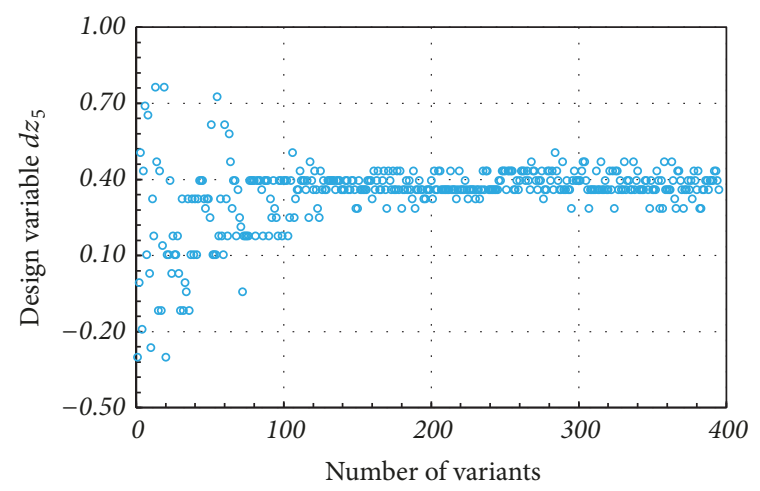

(j)

FIGURE 26: The convergence course of whole speed range optimization for various design variables: (a) $d x_{1}$, (b) $d y_{1}$, (c) $d y_{2}$, (d) $d y_{3}$, (e) $d y_{4}$, (f) $d z_{1}$, (g) $d z_{2}$, (h) $d z_{3}$, (i) $d z_{4}$, and (j) $d z_{5}$.

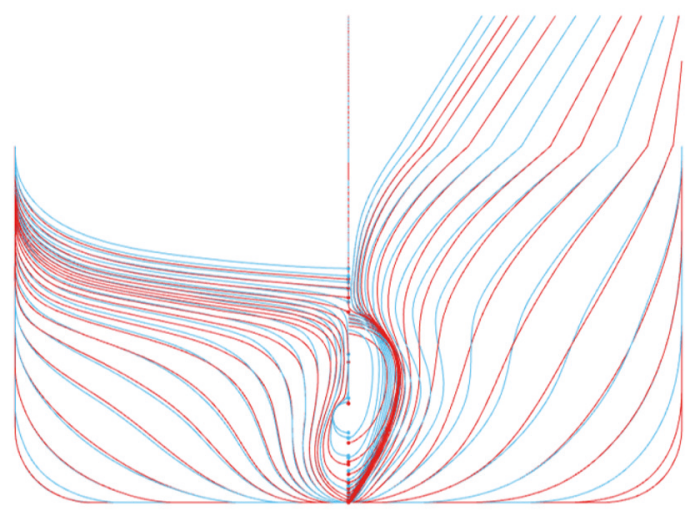

(a)

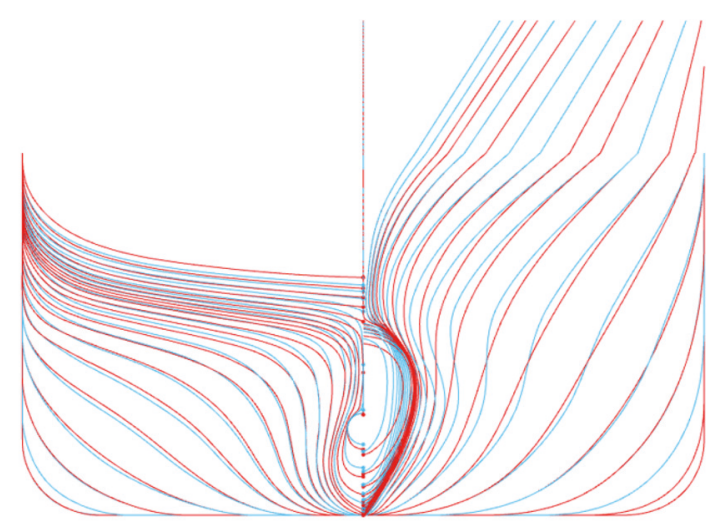

(b)

Figure 27: Comparison of the hull forms: (a) optimized hull form Opt01 (blue) and original hull form (red); (b) optimized hull form Opt02 (blue) and original hull form (red).

stretch forward and fullness is reduced. The upper end of the transition region of the hull is gradually recessed, while the lower end connecting with bulbous bow is gradually protruding outwards. At the same time, the aft ends of Opt01 and Opt02 translate along the $\mathrm{Z}$ axis positive and the overall shape becomes more slender. The total resistance of the optimized hulls based on different optimization algorithms and original hull at various Froude numbers are calculated by the method mentioned above and listed in Table 4 .

The total resistance of the optimized hulls based on different optimization algorithms is all less than that of original hull at various Froude numbers smaller than 0.26. The resistance reduction effect of the optimal scheme OptS01 based on design speed is better than that of the two optimal schemes Opt01and Opt02 based on all speed range. But at the nondesign speed points, the resistance reduction effects of OptS01 are obviously worse than those of Opt01 and Opt02, and as the speed decreases the situation is more obvious.

The wave contours are compared for the optimal scheme Opt01 and original hulls for multiple Froude numbers on the left row in Figure 28. Correspondingly, on the right row in Figure 28, the wave contours are compared for the optimal scheme Opt02 and original hulls for multiple Froude numbers.

In Figure 28, it can be seen that when the Froude number is small, the first peak wave maximum amplitudes of the optimized hull are obviously smaller than those of the original hull. With the increase of Froude number, the contrast difference is gradually reduced. When $F n=0.26$ and more, the first peak wave moves backward in the vicinity of the bow. At the same time, the tail wave amplitudes of the optimized hull form are obviously smaller than those of the original hull under multiple Froude numbers. The optimal schemes Opt01 and Opt02 have similar law of comparison, and the difference is not significant.

In summary, despite the comparison of the total drag coefficient, the comparison of the surface wave shows that the resistance performance of the two optimization schemes is better than that of the original hull. At the same time, it is verified that the resistance optimization design methodology for bow and stern line considering whole speeds range is feasible and reliable. 


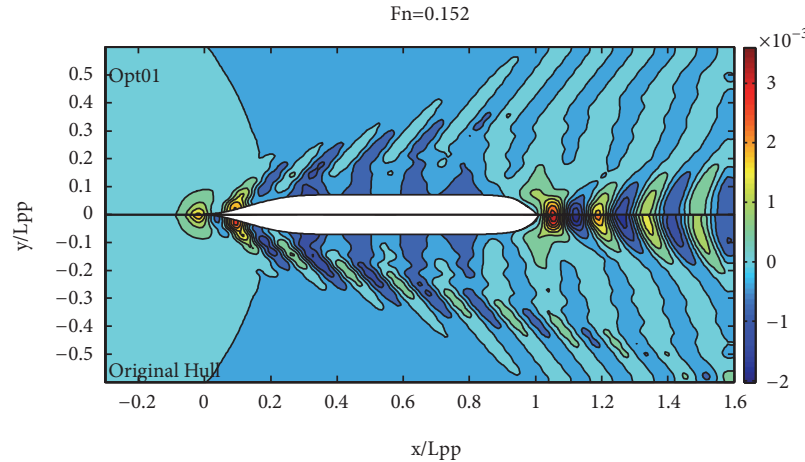

(a1)

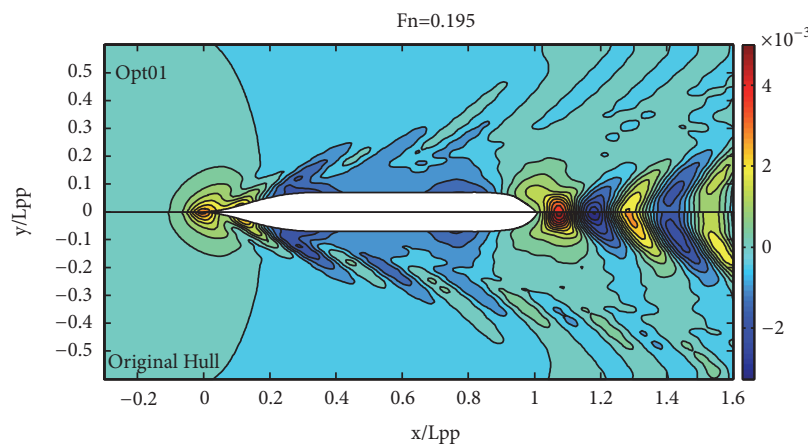

(b1)

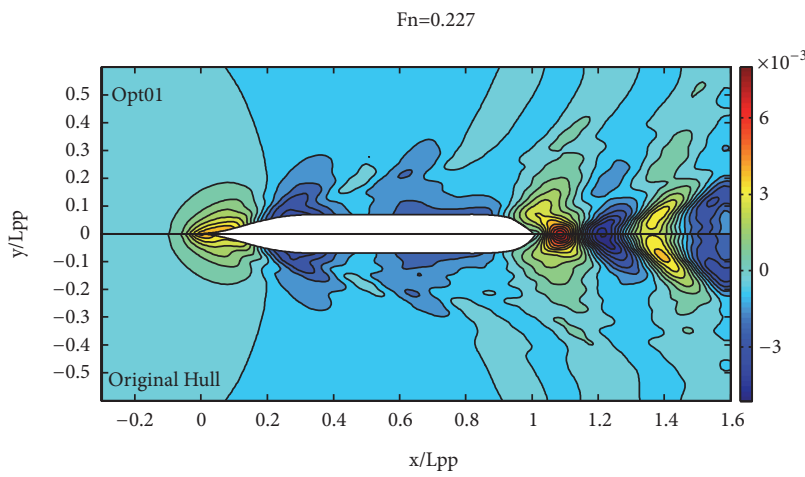

(c1)

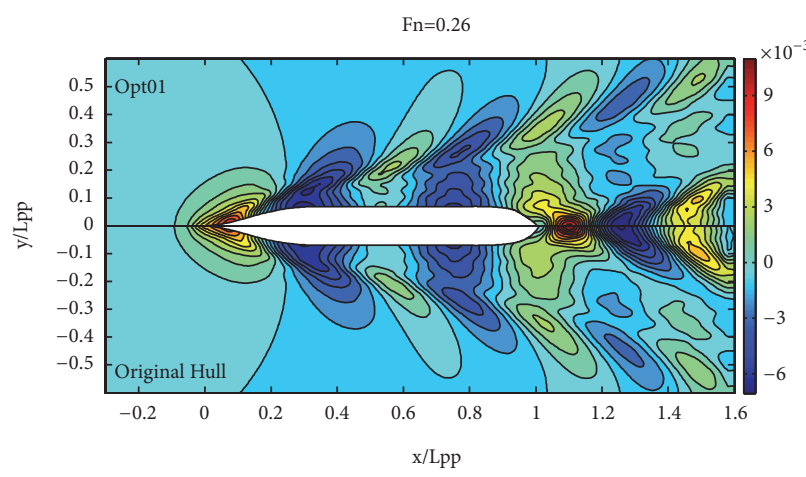

(d1)

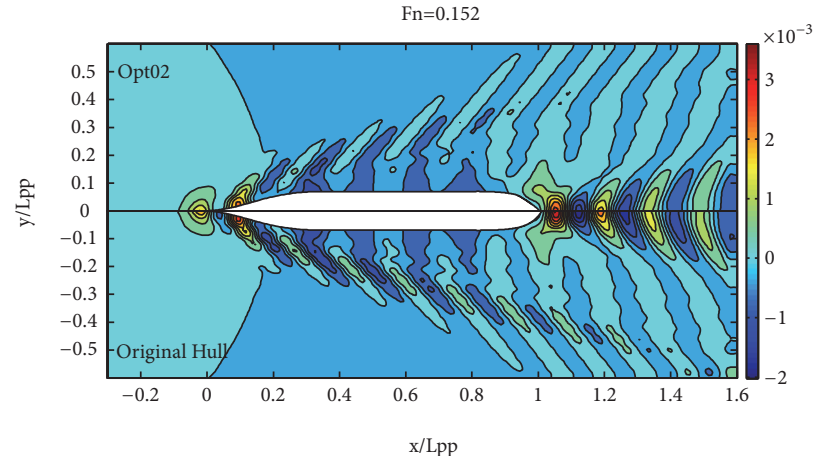

(a2)

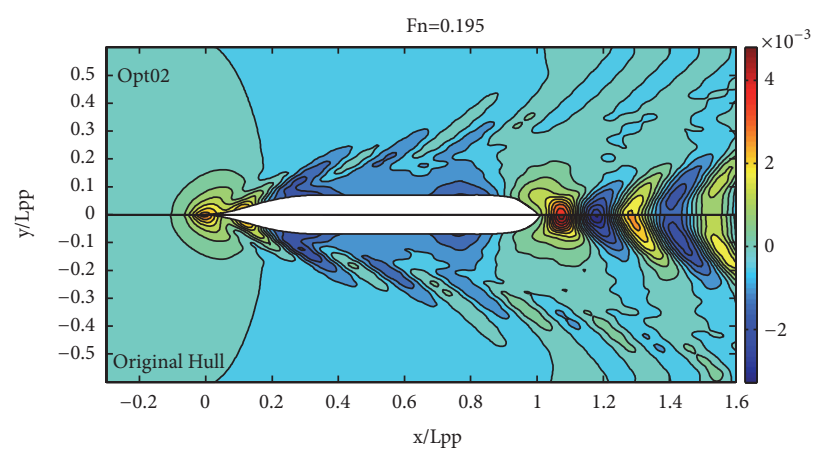

(b2)

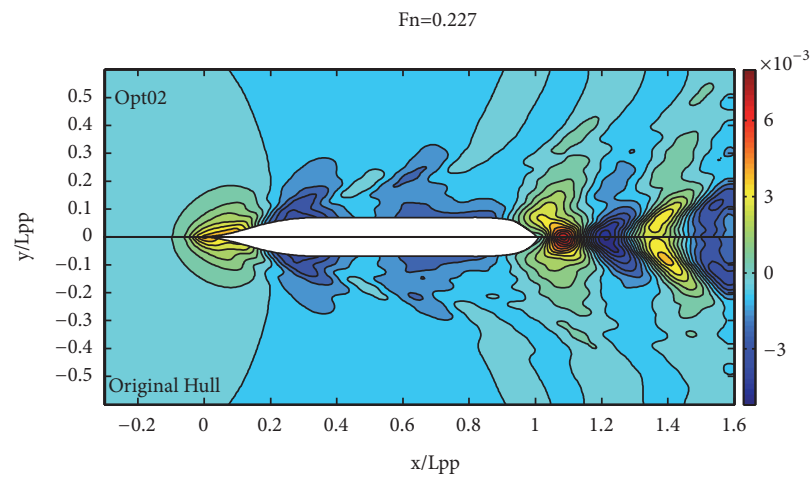

(c2)

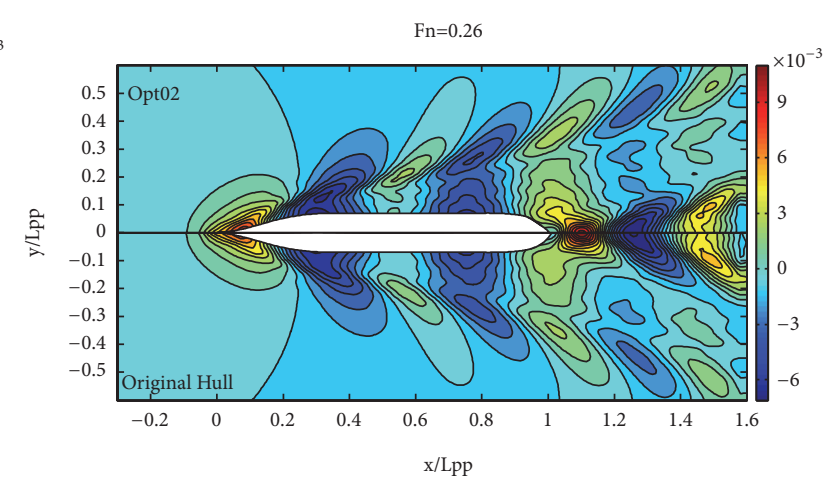

(d2)

FIGURE 28: Continued. 


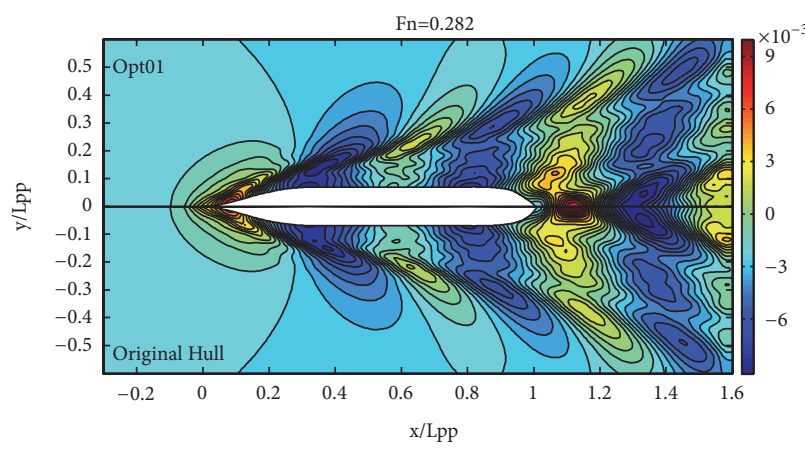

(e1)

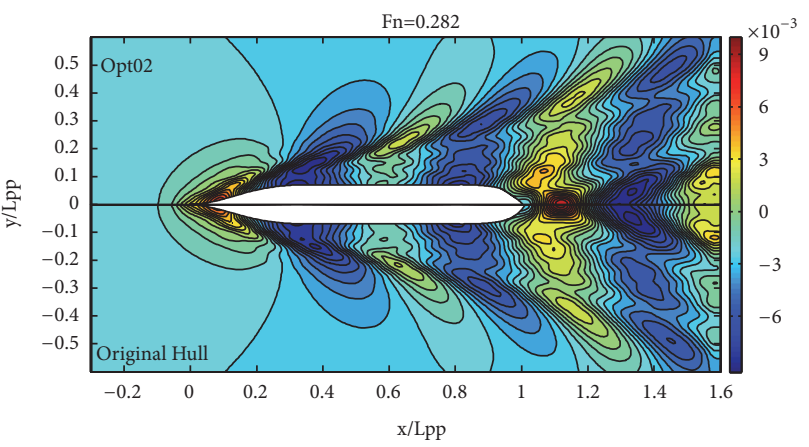

(e2)

Figure 28: Comparison of wave patterns at various Froude numbers: (a1) the optimal scheme Opt01 and original hull at $F n=0.152 ;$ (a2) the optimal scheme Opt02 and original hull at $F n=0.152$; (b1) the optimal scheme Opt01 and original hull at Fn=0.195; (b2) the optimal scheme Opt02 and original hull at $F n=0.195 ;(\mathrm{c} 1)$ the optimal scheme Opt01 and original hull at $F n=0.227$; (c2) the optimal scheme Opt02 and original hull at $F n=0.227$; (d1) the optimal scheme Opt01 and original hull at $F n=0.26$; (d2) the optimal scheme Opt02 and original hull at $F n=0.26$; (e1) the optimal scheme Opt01 and original hull at $F n=0.282$; (e2) the optimal scheme Opt02 and original hull at $F n=0.282$.

TABLE 3: The specific presentations of the optimums Opt01 (left) and Opt02 (right).

\begin{tabular}{|c|c|c|c|c|c|c|c|c|c|c|c|}
\hline $\mathrm{F} 1$ & $95.97 \%$ & & & & & $\mathrm{~F} 1$ & $93.77 \%$ & & & & \\
\hline $\mathrm{F} 2$ & $94.93 \%$ & $\mathrm{dx} 1$ & 1.3 & $\mathrm{dzl}$ & 0.2 & $\mathrm{~F} 2$ & $95.11 \%$ & $\mathrm{dxl}$ & 1.7 & $\mathrm{dzl}$ & 0.3 \\
\hline F3 & $95.23 \%$ & dyl & -1.8 & $\mathrm{dz} 2$ & 0.2 & F3 & $95.14 \%$ & dyl & -2.1 & $\mathrm{dz2}$ & 0.1 \\
\hline $\mathrm{F} 4$ & $95.53 \%$ & dy2 & 171 & dz3 & -0.24 & $\mathrm{~F} 4$ & $95.79 \%$ & dy 2 & 172 & $\mathrm{dz} 3$ & -0.3 \\
\hline F5 & $99.52 \%$ & dy3 & 0.64 & $\mathrm{dz} 4$ & -0.27 & F5 & $99.57 \%$ & dy3 & 0.535 & $\mathrm{dz} 4$ & -0.36 \\
\hline$\left|\nabla / \nabla_{0}-1\right|$ & $0.14 \%$ & dy 4 & -0.49 & $\mathrm{dz} 5$ & 0.397 & $\left|\nabla / \nabla_{0}-1\right|$ & $0.12 \%$ & dy 4 & -0.58 & dz5 & 0.397 \\
\hline$\left|S / S_{0}-1\right|$ & $0.85 \%$ & & & & & $\left|S / S_{0}-1\right|$ & $0.78 \%$ & & & & \\
\hline
\end{tabular}

TABLE 4: Comparison of total resistance between the optimized hulls based on different optimization algorithms and original hull at various Froude numbers.

\begin{tabular}{lccccccc}
\hline & KCS & \multicolumn{2}{c}{ Opt01 } & \multicolumn{2}{c}{ Opt02 } & \multicolumn{2}{c}{ OptS01 } \\
Fn & $\mathrm{Ct}_{0}\left(\times 10^{3}\right)$ & $\mathrm{Ct}\left(\times 10^{3}\right)$ & Reduction & $\mathrm{Ct}\left(\times 10^{3}\right)$ & Reduction & $\mathrm{Ct}\left(\times 10^{3}\right)$ & $\mathrm{Reduction}$ \\
\hline 0.152 & 3.697 & 3.548 & $4.03 \%$ & 3.466 & $6.23 \%$ & 3.598 \\
0.195 & 3.549 & 3.369 & $5.07 \%$ & 3.376 & $4.89 \%$ & 3.377 \\
0.227 & 3.487 & 3.321 & $4.77 \%$ & 3.318 & $4.86 \%$ & 3.338 & $3.85 \%$ \\
0.260 & 3.653 & 3.490 & $4.47 \%$ & 3.499 & $4.21 \%$ & 3.378 \\
0.282 & 4.489 & 4.467 & $0.48 \%$ & 4.469 & $0.43 \%$ & 4.499 \\
\hline
\end{tabular}

\section{Conclusions}

A resistance optimization design methodology for bow and stern line considering all range of speeds has been implemented and applied. Firstly, the partially parametric mapping deformation method is presented via the features parametric curve. Accordingly the deformation theory formula has been put forward for launching the three-dimensional partial parametric hull deformation and geometry smoothing transition. Secondly, the method for evaluating the ship total resistance is proposed by combining the ITTC 1957 modelship correlation formula with the potential flow Rankine source panel method. By this method, the total resistance of KCS container ship is calculated which agrees well with the corresponding experimental data and further acquires validation with the overall error of $2.0 \%$.
Accordingly the ship bow and stern of KCS have been optimized under conditions of the single design speed. The optimization results show a decrease of $7.0 \%$ in the total resistance at the design speed of $\mathrm{Fn}=0.26$, but the resistance reduction benefit is weakened at other speed points. For example, at low speed of $\mathrm{Fn}=0.152$, the drag reduction benefit is reduced to about $2 \%$, and at high speed of $\mathrm{Fn}=0.282$ the resistance is slightly increased. The reason of drag reduction is analyzed through the comparison of wave profiles for the original and optimized hulls.

Then the ship bow and stern of KCS have been optimized under conditions of whole speeds range and two optimal schemes are obtained. The optimization results are comprehensively analyzed by comparing the total resistance and the wave profiles between the original and optimized hulls. Through the analysis, it can be seen that when the Froude 
number is in $0.152-0.26$, the drag reduction benefits of the two optimization schemes at each speed point all averagely remain above $4.0 \%$ of ship resistance decrease, some as high as $6 \%$. At high speed of $F n=0.282$, the drag reduction benefit has some drop.

Overall, the drag reduction benefits of the optimal scheme based on whole speed range are not as good as those based on the design speed, but more balanced. Especially for modern container ships, its operating condition is often not fixed. In order to save energy, the measures of reducing speed are often adopted, and the ships rarely sail at design speed. Therefore, while there is no special requirement or definite limit, the resistance optimization design methodology considering whole speeds range present in this paper can provide some guidance in early stages of ship design.

In conclusion, the present study shows an effective approach for the resistance optimization of modern container ships design which considers whole speed range and offers constructive assistance for designers who are attempting to obtain superior resistance performance through optimized designs.

\section{Data Availability}

The data used to support the findings of this study are available from the corresponding author upon request.

\section{Conflicts of Interest}

The authors declare no conflicts of interest.

\section{Acknowledgments}

The authors thank the reviewers for their comments and suggestions in improving the quality of the article. This work was funded by the National Natural Science Foundation of China (Grant No. 51809029), the Ph.D. Scientific Research Fund of the Natural Science Foundation of Liaoning Province (Acceptance No. 1552440867180), the Polar Shipping and Safety Research Institute of China, DMU (Grant No. 3132019306), and the Fundamental Research Funds for the Central Universities under Grant Nos. 3132019113 and 3132018207.

\section{References}

[1] C. Banks, O. Turan, A. Incecik et al., "Understanding ship operating profiles with an aim to improve energy efficient ship operations," in Proceedings of the Low Carbon Shipping Conference, pp. 9-10, London, UK, 2013.

[2] H. Lackenby, "On the systematic geometrical variation of ship form," Trans INA, vol. 92, pp. 289-316, 1950.

[3] S. Harries, Parametric design and hydrodynamic optimization of ship hull forms [Ph.D.Thesis], Institut fur Schiffs-und Meerestechnik, Technische Universitat Berlin, Germany, 1998.

[4] S. Harries and C. Abt, "Parametric curve design applying fairness criteria," in Proceedings of the International Workshop on Creating Fair and Shape-Preserving Curves and Surfaces, 1998.
[5] H. Nowacki and E. D. Horst, "Creating fair and shape preserving curves and surfaces," in Proceedings of the International Workshop Organized by the EU Network Fairshape, Kleinmachnow, 1997.

[6] S. Harries and H. Nowacki, "Form parameter approach to the design of fair hull shapes," in Proceedings of the 10th International Conference on Computer Applications, ICCAS, MIT, Cambridge, MA, USA, 1999.

[7] Y. S. Lee, Trends validation of CFD predictions for ship design purpose [Ph.D. thesis], Institut fur Schiffs-und Meerestechnik, Technische Universitat Berlin, Germany, 2003.

[8] J. J. Maisonneuve, S. Harries, J. Marzi, H. C. Raven, U. Viviani, and H. Piippo, "Toward optimal design of ship hull shapes," in Proceedings of the 8th international marine design conference, IMDC03, pp. 31-42, Athens, 2003.

[9] G. K. Saha, K. Suzuki, and H. Kai, "Hydrodynamic optimization of ship hull forms in shallow water," Journal of Marine Science and Technology, vol. 9, no. 2, pp. 51-62, 2004.

[10] G. K. Saha, K. Suzuki, and H. Kai, "Hydrodynamic optimization of a catamaran hull with large bow and stern bulbs installed on the center plane of the catamaran," Journal of Marine Science and Technology, vol. 10, no. 1, pp. 32-40, 2005.

[11] H. C. Kim, Parametric design of ship hull forms with a complex multiple domain surface topology [Ph.D. thesis], University of Berlin, Germany, 2004.

[12] F. Pérez and J. A. Clemente, "Constrained design of simple ship hulls with B-spline surfaces," Computer-Aided Design, vol. 43, no. 12, pp. 1829-1840, 2011.

[13] F. Pérez, J. A. Suárez, J. A. Clemente, and A. Souto, "Geometric modelling of bulbous bows with the use of non-uniform rational B-spline surfaces," Journal of Marine Science and Technology, vol. 12, no. 2, pp. 83-94, 2007.

[14] F. Pérez-Arribas, J. A. Suárez-Suárez, and L. FernándezJambrina, "Automatic surface modelling of a ship hull," Computer-Aided Design, vol. 38, no. 6, pp. 584-594, 2006.

[15] C. Abt and S. Harries, "A New Approach to Integration of CAD and CFD for Naval Architects", in Proceedings of the 6th International Conference on Computer Applications and Information Technology in Maritime Industries, COMPIT, Cortona, Italy, 2007.

[16] S. Harries, "Serious play in ship design, tradition and future of ship design in Berlin," Tech. Rep., Technical University of Berlin, 2008.

[17] P. Zhang, D. Zhu, and W. Leng, "Parametric approach to design of hull forms," Journal of Hydrodynamics, vol. 20, no. 6, pp. 804810, 2008.

[18] Y. Tahara, D. Peri, E. F. Campana, and F. Stern, "Computational fluid dynamics-based multiobjective optimization of a surface combatant using a global optimization method," Journal of Marine Science and Technology, vol. 13, no. 2, pp. 95-116, 2008.

[19] A. I. Ginnis, C. Feurer, K. A. Belibassakis et al., "A CATIA ${ }^{\circledR}$ ship-parametric model for isogeometric hull optimization with respect to wave resistance," in Proceedings of the International Conference on Computer Applications in Shipbuilding 2011, vol. 1, pp. 9-20, Italy, 2011.

[20] A. I. Ginnis, R. Duvigneau, C. Politis et al., "A multi-objective optimization environment for Ship-Hull Design based on a BEM-Isogeometric solver," in Proceedings of the 5th Conference on Computational Methods in Marine Engineering, Springer, Hamburg, Germany, 2013. 
[21] A. Ginnis, K. Kostas, C. Politis et al., "Isogeometric boundaryelement analysis for the wave-resistance problem using Tsplines," Computer Methods Applied Mechanics and Engineering, vol. 279, pp. 425-439, 2014.

[22] K. Kostas, A. Ginnis, C. Politis, and P. Kaklis, "Ship-hull shape optimization with a T-spline based BEM-isogeometric solver," Computer Methods Applied Mechanics and Engineering, vol. 284, pp. 611-622, 2015.

[23] C. Abt, S. Harries, J. Heimann, and H. Winter, "From redesign to optimal hull line by means of parametric modeling," in Proceedings of the 2nd International on Computer Applications and Information Technology in the Marine Industries, pp. 444458, Hamburg, Germany, 2003.

[24] Y. Lu, X. Chang, and A. Hu, "A hydrodynamic optimization design methodology for a ship bulbous bow under multiple operating conditions," Engineering Applications of Computational Fluid Mechanics, vol. 10, no. 1, pp. 330-345, 2016.

[25] T. W. Lowe and J. Steel, "Conceptual hull design using a genetic algorithm," Journal of Ship Research, vol. 47, no. 3, pp. 222-236, 2003.

[26] K. Suzuki, H. Kai, and S. Kashiwabara, "Studies on the optimization of stern hull form based on a potential flow solver," Journal of Marine Science and Technology, vol. 10, no. 2, pp. 61-69, 2005.

[27] B. Zhang, K. Ma, and Z. Ji, “The optimization of the hull form with the minimum wave making resistance based on rankine source method," Journal of Hydrodynamics, vol. 21, no. 2, pp. 277-284, 2009.

[28] B. J. Zhang, "Research on optimization of hull linesfor minimum resistance based on Rankine source method," Journal of Marine Science and Technology, vol. 20, no. 1, pp. 89-94, 2012.

[29] C. Yang, F. Huang, and F. Noblesse, "Practical evaluation of the drag of a ship for design and optimization," Journal of Hydrodynamics, vol. 25, no. 5, pp. 645-654, 2013.

[30] H. J. Choi, D. W. Park, and M. S. Choi, "Study on optimized hull formof basic ships using optimization algorithm," Journal of Marine Science and Technology - Taiwan, vol. 23, no. 1, pp. 60-68, 2015.

[31] C. Yang, F. Huang, and H. Kim, "Hydrodynamic optimization of a triswach," Journal of Hydrodynamics, vol. 26, no. 6, pp. 856864, 2014.

[32] J. H. Holland, Adaptation in Natural and Artificial Systems: An Introductory Analysis with Applications to Biology, Control, and Artificial Intelligence, MIT Press, 1992.

[33] K. Deb, S. Agrawal, A. Pratap, and T. Meyarivan, "A fast elitist non-dominated sorting genetic algorithm for multi-objective optimization: NSGA-II," in Proceedings of the International Conference on Parallel Problem Solving From Nature, vol. 1917, pp. 849-858, Springer.

[34] K. Deb, A. Pratap, S. Agarwal, and T. Meyarivan, "A fast and elitist multiobjective genetic algorithm: NSGA-II," IEEE Transactions on Evolutionary Computation, vol. 6, no. 2, pp. 182197, 2002.

[35] L. Larsson, F. Stern, and V. Bertram, "Benchmarking of computational fluid dynamics for ship flows: The Gothenburg 2000 workshop," Journal of Ship Research, vol. 47, no. 1, pp. 63-81, 2003.

[36] T. Hino, Proceedings of CFD Workshop Tokyo 2005, National Maritime Research Institute, Tokyo, Japan, 2005.

[37] L. Larsson, F. Stern, and M. Visonneau, Numerical Ship Hydrodynamics-An assessment of the Gothenburg 2010 Workshop, Springer Science \& Business Media, Netherlands, 2014. 


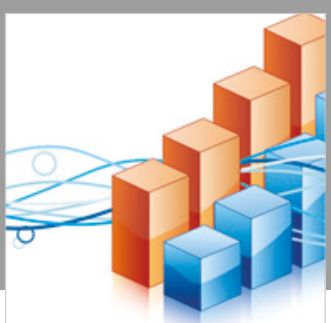

Advances in

Operations Research

\section{-n-m}
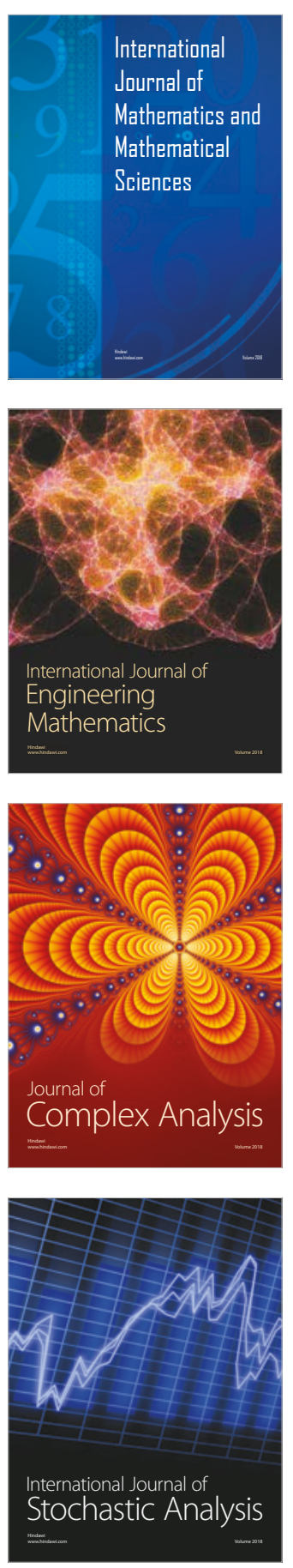
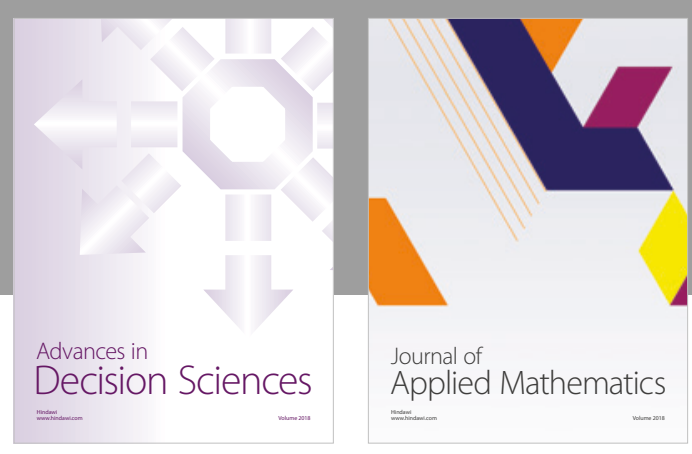

Journal of

Applied Mathematics
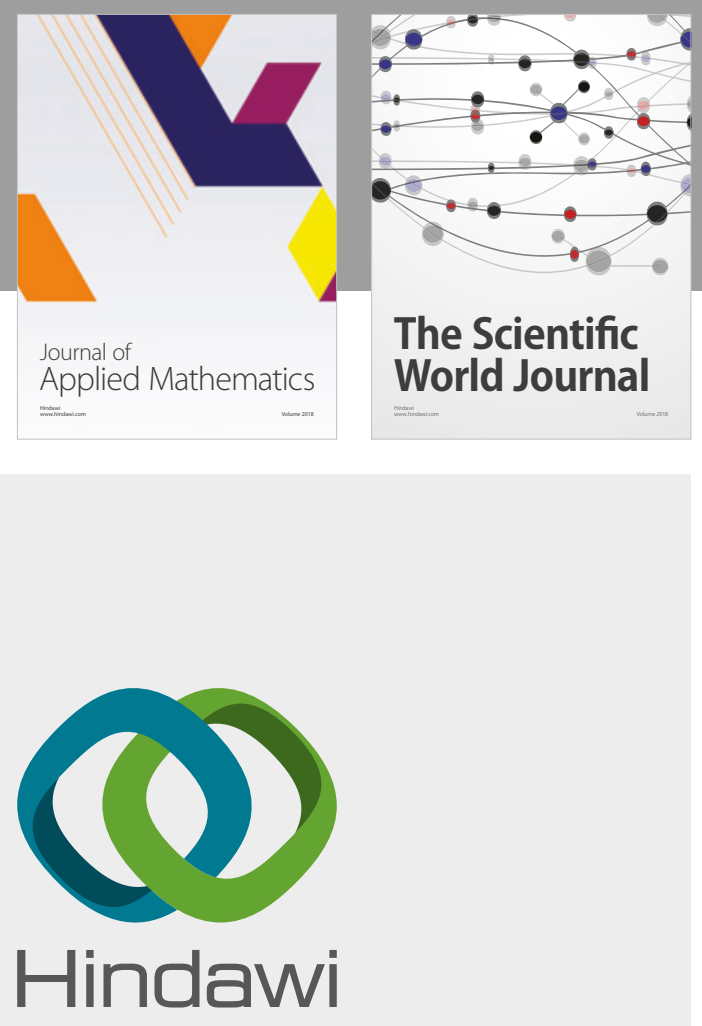

Submit your manuscripts at

www.hindawi.com

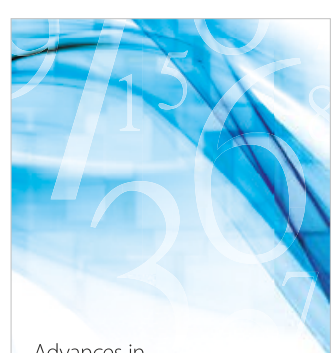

Advances in
Numerical Analysis
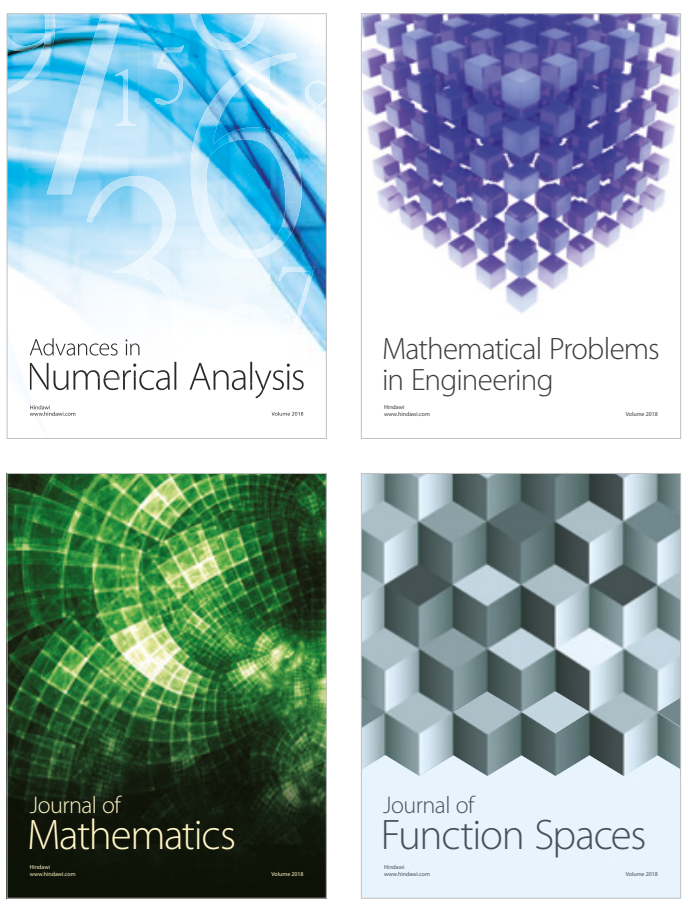

Mathematical Problems in Engineering

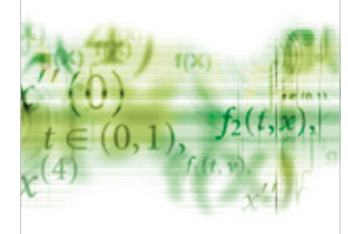

International Journal of

Differential Equations

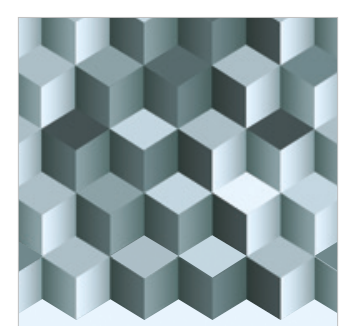

Journal of

Function Spaces

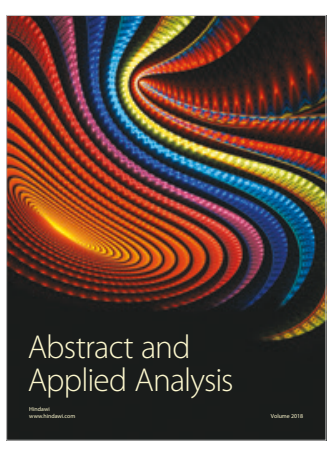

The Scientific

World Journal

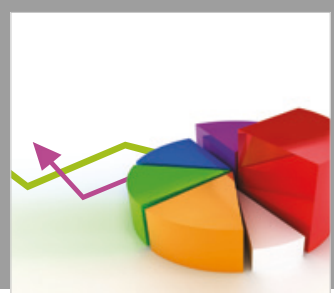

Journal of

Probability and Statistics
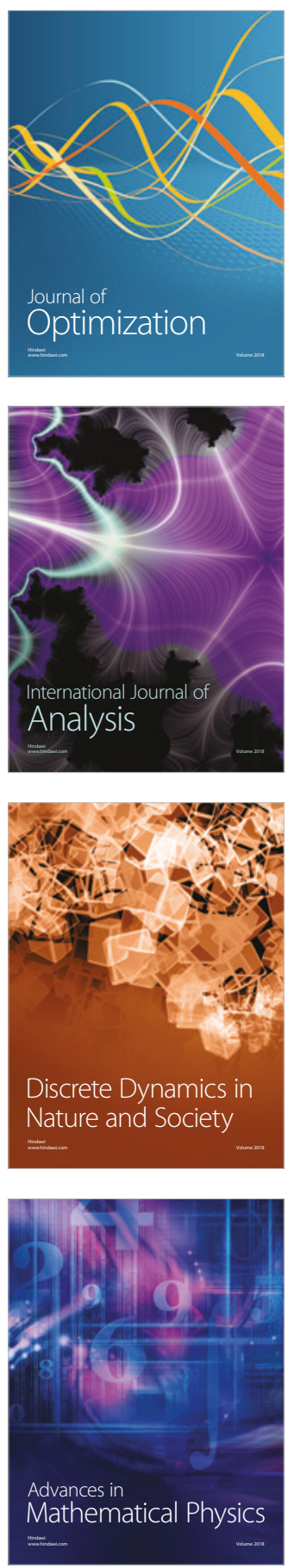